OPEN ACCESS

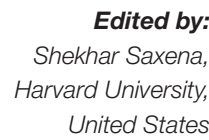

Reviewed by:

Jana Chihai,

Nicolae Testemiţanu State University of Medicine and Pharmacy,

Moldova

Sarah Skeen,

Stellenbosch University,

South Africa

*Correspondence:

Keng-Yen Huang

keng-yen.huang@nyulangone.org

Specialty section:

This article was submitted to

Public Mental Health,

a section of the journal

Frontiers in Psychiatry

Received: 28 May 2019 Accepted: 11 October 2019

Published: 13 November 2019

Citation:

Huang K-Y, Lee D, Nakigudde J,

Cheng S, Gouley KK, Mann D,

Schoenthaler A, Chokshi S,

Kisakye EN, Tusiime $C$ and

Mendelsohn A (2019) Use of

Technology to Promote Child Behavioral Health in the Context of

Pediatric Care: A Scoping Review

and Applications to Low- and

Middle-Income Countries.

Front. Psychiatry 10:806.

doi: 10.3389/fpsyt.2019.00806

\section{Use of Technology to Promote Child Behavioral Health in the Context of Pediatric Care: A Scoping Review and Applications to Low- and Middle-Income Countries}

Keng-Yen Huang ${ }^{1 *}$, Douglas Lee ${ }^{2}$, Janet Nakigudde ${ }^{3}$, Sabrina Cheng ${ }^{1}$, Kathleen Kiely Gouley ${ }^{1}$, Devin Mann ${ }^{1}$, Antoinette Schoenthaler ${ }^{1}$, Sara Chokshi', Elizabeth Nsamba Kisakye ${ }^{4}$, Christine Tusiime ${ }^{5}$ and Alan Mendelsohn ${ }^{1}$

\begin{abstract}
Department of Population Health, New York University School of Medicine, New York, NY, United States, ${ }^{2}$ College of Osteopathic Medicine, New York Institute of Technology, New York, NY, United States, ${ }^{3}$ Department of Psychiatry, Makerere University, Kampala, Uganda, ${ }^{4}$ Ministry of Education and Sports, Kampala, Uganda, ${ }^{5}$ Butabika Hospital, Kampala, Uganda
\end{abstract}

Background: The burden of mental, neurological, and substance (MNS) disorders is greater in low- and middle-income countries (LMICs). The rapid growth of digital health (i.e., eHealth) approaches offer new solutions for transforming pediatric mental health services and have the potential to address multiple resource and system barriers. However, little work has been done in applying eHealth to promote young children's mental health in LMICs. It is also not clear how eHealth has been and might be applied to translating existing evidence-based practices/strategies (EBPs) to enable broader access to child mental health interventions and services.

Methods: A scoping review was conducted to summarize current eHealth applications and evidence in child mental health. The review focuses on 1) providing an overview of existing eHealth applications, research methods, and effectiveness evidence in child mental health promotion (focused on children of 0-12 years of age) across diverse service contexts; and 2) drawing lessons learned from the existing research about eHealth design strategies and usability data in order to inform future eHealth design in LMICs.

Results: Thirty-two (32) articles fitting our inclusion criteria were reviewed. The child mental health eHealth studies were grouped into three areas: i) eHealth interventions targeting families that promote child and family wellbeing; ii) eHealth for improving school mental health services (e.g., promote school staff's knowledge and management skills); and iii) eHealth for improving behavioral health care in the pediatric care system (e.g., promote use of integrated patient-portal and electronic decision support systems). Most eHealth studies have reported positive impacts. Although most pediatric eHealth studies were conducted in high-income countries, many eHealth design strategies can be adapted and modified to fit LMIC contexts. Most user-engagement strategies identified from high-income countries are also relevant for populations in LMICs. 
Conclusions: This review synthesizes patterns of eHealth use across a spectrum of individual/family and system level of eHealth interventions that can be applied to promote child mental health and strengthen mental health service systems. This review also summarizes critical lessons to guide future eHealth design and delivery models in LMICs. However, more research in testing combinations of eHealth strategies in LMICs is needed.

Keywords: mHealth, eHealth, pediatric, behavioral health, parenting, framework, health service, low-and-middleincome country

\section{BACKGROUND}

\section{Pediatric Mental Health Needs and Service Challenges in LMICs}

The burden of mental, neurological, and substance (MNS) disorders accounts for $10 \%-14 \%$ of the Global Burden of Disease $(1,2)$, and this burden is greater in low-and-middle-income countries (LMICs) because of high rates of poverty, violence, health problems, and inadequate health systems $(3,4)$. An estimated 171 million young children in LMICs are "off track" in behavioral- and social-emotional development, which places them on the path to MNS disorders (5). Early prevention and intervention strategies focusing on child mental and behavioral health promotion strategies can reduce this burden and its sequelae, but limited mental health prevention and treatment services are available for children and families in LMICs. Although the World Health Organization (WHO) recognizes MNS disorders as a global priority, and MNS disorders are now discussed at the highest-level policy forums devoted to global health and development, solutions for reducing burden remain limited.

Population health is largely influenced by social determinants $(6,7)$. High child mental health burden in LMICs may stem from inequalities in social status, resource allocation and opportunities, medical and social service access, and the quality of living environments (7-9). Many children in LMICs are living in families with low financial capital and high levels of family stress (e.g., violence, poverty-related stressors, maltreatment) and in communities with poor mental health service, system, and resource. Adversities and stressors experienced by families can undermine positive parenting and child behavioral regulation, which are associated with higher mental health problems in young children in LMICs $(10,11)$. From a services perspective, children and parents from LMICs are far less likely than families from high-income countries to have access to parenting information, preventive or promotive mental health services, or participate in evidence-based early interventions because of the lack of child mental health resources and systems networks. Therefore, to effectively address children's behavioral and mental health needs, and minimize disparities in LMICs, solutions that focus on a wide range of individual, family, systems, and service determinants as well as prioritizing early prevention and intervention - are needed (12-14).

\section{Rapid Growth of eHealth Offers New Solutions to Address Barriers At Multiple Levels}

The rapid growth and widespread of technology has the potential to address child mental health burden in LMICs by offering new solutions for improving health information and supports, service access, and resource challenges. Emerging studies from both high- and LMICs have provided supporting evidence of the potential to transform health services and systems using eHealth $(15,16)$. eHealth is defined as the use of information and communication technology (ICT), such as computers, mobile phones, communications satellite, patient monitors, and other technology tools for all aspects of health information, services, and integrated systems. mHealth (or mobile-health), a subcomponent of eHealth, is defined as the use of mobile devices (e.g., mobile phones, portable/mobile patient monitoring devices, personal digital assessment devices, and other wireless devices) for individual medical and public health practice (17). eHealth can be tailored to individual needs, provided at low-cost, used to improve distance communication barriers, support training and management, and is a sustainable implementation model $(15,18)$. eHealth has been recommended by the WHO as a health servicestrengthening strategy, and shown to be effective in promoting individual patient health, enhancing family engagement, health knowledge, service access, team communication, and emergency support globally (19).

At the global country-level, since 2005, WHO has initiated the Global Observatory for eHealth (GOe), a joint group effort to support WHO member countries (including LMICs) to adopt digital technologies to improve public health as well as individual health and well-being (20). Much progress has been made in policies and legislation since the establishment of GOe. As indicated in the 2016 report, $87 \%$ of WHO member states had one or more national initiatives on mHealth, $58 \%$ of countries had applied eHealth strategies, and almost all of those (56\%) had initiated eHealth for monitoring and surveillance of maternal, neonatal, and pediatric health $(17,21-23)$.

At the eHealth programming and intervention level, two recent eHealth scoping reviews also summarize progress of eHealth applications in child health promotion and in LMIC contexts. In the eHealth applications for child health, Barros and Greffin's (24) review (24) of 119 technology-based, parentfocused interventions found that different formats of web-based applications have targeted parents and aimed at health-related 
promotion, via both prevention and treatment support (e.g., universal preventions and interventions focused on adaptation to and management of chronic/severe acute health conditions). Most of these e-parent interventions were adapted from evidencebased interventions (EBIs), focused on child physical health (i.e., obesity, healthy eating, vaccination, child safety, alcohol/substance use, health service use, oral health, sexual, and reproductive health), and based on studies from high-income countries. The pediatric eHealth Strategies used were also focused on promoting parental self-management, specific parenting skills, or parent support (e.g., social support, providing customized feedback) (24). In the eHealth applications in the LMIC contexts, Bervell and Al-Samarraie (25) reviewed 66 articles to understand patterns of eHealth use across a spectrum of disease and health conditions in Sub-Saharan African (SSA) countries. They found that eHealth has been applied in a range of diseases/health conditions in SSA, including tropical diseases, infectious diseases (malaria, HIV/ AIDS), oral health, infant health-related conditions, maternal health-related conditions (antenatal/postnatal care, postpartum hemorrhage), noncommunicable diseases (cervical cancer, blood pressure), and mental health (depression care). In addition, most eHealth strategies were designed for the purposes of disease/ condition control and prevention (e.g., reminders toward medical care/activity adherence), population health monitoring and case report, information provision for treatment/prevention (e.g., health information to patients or health workers), data acquisition and patient records management, diagnosis (telepathology, digital radiology tools), training/recruiting/retaining health professionals, or decision-making/referrals (25).

While many eHealth benefits have been reported in the literature to date, there are limitations as well. For example, eHealth has been shown to be more useful for addressing lowintensity, high-frequency behavioral difficulties than highintensity behavioral difficulties (26). For users with low literacy (e.g., low digital/technology and/or low literacy/educational attainment) or systems with low eHealth technology capacity or resource availability, the limitations would be greater. Additional steps would be needed before applying eHealth in these contexts $(27,28)$. Furthermore, the benefits of eHealth may not be guaranteed because mixed results are often reported (29). Well-designed, high quality evaluations are needed to better understand the factors and service delivery approaches and contexts that that contribute to of eHealth-related benefits (30).

In sum, the literature thus far suggests that much progress has been made in the development and implementation of eHealth strategies in both child health promotion and in LMICs; however, applications of these strategies in early childhood behavioral and mental health in LMICs remain limited. Given the growth and spread of technology and access, especially given the explosion in digital device ownership and improvements of ICT systems in LMICs (45\%-89\% with mobile-cellular telephone, 45\%-54\% with smartphone, $7 \%-18 \%$ with internet access) $(20,23,31$, 32 ), and the potential of eHealth to address multiple resource and system management barriers, an effort to build on existing evidence and develop new strategies for child mental health promotion is needed.

\section{The Study Aims}

As the first step to inform the development of eHealth (including mHealth) interventions and services for child mental health promotion in LMIC contexts, it is critical to understand and summarize current research and knowledge, especially related to the technology solutions/strategies, core components, and evidence that contribute to effective child mental health promotion. Moreover, to effectively reduce population mental health burden, early prevention and intervention eHealth strategies in children need to be prioritized. Thus, the overall goal of this paper is to address these eHealth knowledge gaps by reviewing related eHealth literature and applications focused on young children. This scoping review focuses specifically on:

1. Providing an overview of existing eHealth applications, research methods, and evidence of effectiveness in child mental health promotion (focused on children of $0-12$ years of age) in diverse service contexts.

2. Drawing lessons learned from the existing research about the design strategies to promote usage (or user-engagement in technology use) and evidence of usability, acceptability, and satisfaction (usage patterns, level of engagement, and satisfaction in usage) to inform design, delivery, and evaluation strategies of future eHealth interventions in LMIC contexts.

\section{METHODS}

\section{Literature Review Methods}

A scoping review, drawing upon a broad range of applications of technology in medicine, psychology, and pediatric-related literature, was conducted. The scoping review method was applied because it provides a useful initial approach to generate foundational knowledge, and to inform approaches for a future systematic review (33). Therefore, this paper was not intended to be an exhaustive review of the literature, but rather to provide a high-level view of the approaches to the use and evaluation of eHealth strategies for child mental health promotion and prevention. In our scoping review, the five-step method outlined by Arksey and O'Malley (33) was applied. The five steps include: (1) identifying the research question; (2) identifying relevant studies/literature; (3) selecting studies; (4) charting the data; and (5) collating, summarizing, and reporting results.

A comprehensive literature search using the PubMed and PsycInfo databases was undertaken. Literature search terms used in this review are detailed in Box 1 in the Appendix Supplemental File. Included papers were critically appraised using the Preferred Reporting Items for Systematic Reviews and Meta-Analyses-Extension for Scoping Review (PRIMSMA-ScR) guideline (34). The overall inclusion criteria of articles for this review included studies that: (1) examined eHealth applications in pediatric behavioral and mental health promotion or intervention; (2) reported either intermediate impacts (e.g., on providers) or direct impacts on child well-being; (3) examined eHealth strategies used in diverse service contexts (i.e., primary care, school, and community contexts); (4) were focused on young children (birth to 12 years; not adolescents); (5) were peer 
reviewed, published in English, in PubMed or PsycInfo from 2010 to 2018 ; (6) were not using telehealth or messaging/texting; and (7) were not focused on behavioral health related to childhood obesity and substance/alcohol use. We did not include telehealth in the review because most families in LMICs do not have access to digital tools for videoconferencing, which has been reported to be a more effective child telemental health approach (35). We did not include messaging/texting in the review because evidence suggests effective public health approaches to child mental health intervention require consideration of multicomponent interventions (e.g., including multiple domains of mental health knowledge, skill training, and practice support in parenting/child/health-worker interventions) (36-38). However, existing messaging/texting strategies tend to be used as support or enhancement strategies for interventions, and might be limited in serving as stand-alone mental health intervention strategies in multicomponent interventions (39-41). Finally, many adults and parents in LMICs have low literacy (primary or less than primary school education) and do not have smartphones, which make a messaging approach challenging. Figure 1 shows the flowchart diagram of the selection of articles. Because this review relied on publicly available documents and, therefore, was exempt from Institutional Review Board determination.

\section{RESULTS}

One-hundred-and-forty peer reviewed articles from PubMed and 40 from PsycInfo were identified. Two duplicated articles were excluded. After reviewing for appropriateness, 32 articles (from 30 eHealth studies) were included for final review. Of these, 2 were scoping reviews, 4 were protocol papers (all using a randomized controlled trial design), and 26 were empirical articles that used a range of designs (i.e., experimental, prepost comparison, cross-sectional, qualitative, or mixed method designs), and in different phases of eHealth design and testing (e.g., early user-design, feasibility, or efficacy testing phases). Table 1S in the Supplemental file documents the charting of review data in detail for the included studies.

Across the articles reviewed, three broad categories of literature about eHealth in child mental health emerged. We synthesized knowledge and lessons learned separately for these three areas: i) eHealth interventions targeting family members; ii) eHealth for improving school mental health services; and iii) eHealth for improving behavioral health service in primary care settings. About half of the eHealth studies (16/30) were conducted in the US, only one study was conducted in LMIC (Brazil), and the remaining studies were conducted in developed countries (13/30, such as Australia (5/30), UK, New Zealand, Netherlands). Regarding the content of eHealth interventions, about half of the eHealth studies (17/30) were adapted from evidence-based interventions (EBIs). The majority of the studies (23/30) reported impacts of the intervention using an experimental design (14/30), and almost all of these studies reported significant positive impacts $(21 / 23)$.

Figure 2 provides a pictorial view that summarizes strategies and ways that eHealth has been applied in these three areas.

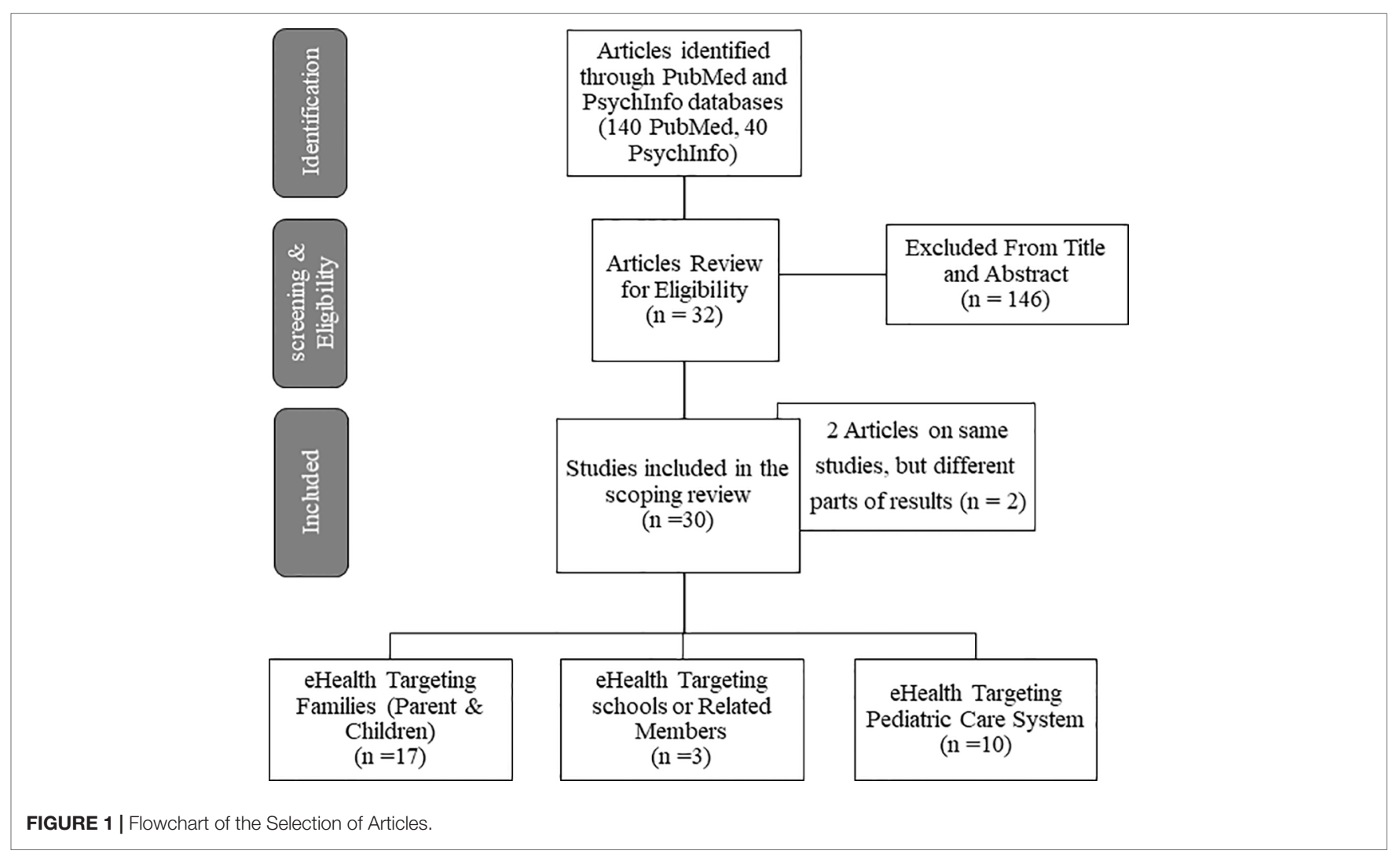




\begin{tabular}{|c|}
\hline $\begin{array}{l}\text { A. efiealth Targeting Families (Parents \& } \\
\text { Children) } \\
\text { (71\% based on EBIs) }\end{array}$ \\
\hline $\begin{array}{l}\text { Parenting Interventions } \\
\text { (Universal or targeted for parenting } \\
\text { promotion or support) }\end{array}$ \\
\hline $\begin{array}{l}\text { a. Web \& indivicual-based parenting } \\
\text { sessions only (EXP) }(1,6,8,12)\end{array}$ \\
\hline $\begin{array}{l}\text { Web \& individual-based parenting } \\
\text { with communication support (phone } \\
\text { coaching, reminder, feedback } \\
\text { message, email, social media group) } \\
\text { (EXP) }(3,4,7,10,11,13,14) \\
\text { Social-media group-based parent }\end{array}$ \\
\hline $\begin{array}{l}\text { support sessions (e.g., Facebook) } \\
\text { (Review) (5) }\end{array}$ \\
\hline $\begin{array}{l}\text { In-person sessions } \div \text { social mediz } \\
\text { group-based support (EXP, Non- } \\
\text { EXP) }(2,9)\end{array}$ \\
\hline Child-focused Interrentions \\
\hline a. Games-individual based (EXP)(15) \\
\hline $\begin{array}{l}\text { b. Peer support group (exly } \\
\text { development)(16) }\end{array}$ \\
\hline $\begin{array}{l}\text { Combination of parenting }+ \text { child focused } \\
\text { intervention }+ \text { Conmunim tion support }\end{array}$ \\
\hline $\begin{array}{l}\text { 3. Individual-based eCBT for } \\
\text { childhood anxiety (Non-EXP) (17) }\end{array}$ \\
\hline
\end{tabular}

\section{B. effealth Targeting Schools or} RelatedMembers (33\% based on BBIs)

eLearning for teachers to promote child mental health knowledge and classioom behavioml management

2. Web-education video $\div$ web-based discussion forum + web conference + support text for teachers in LMICs (EXP) (18)

eHealth tools to support teachers and students in managing and promoting behaviomal regulation in child ren

a. Tablet prompts child behavicral regulation (self-evaluation + incentive strategies) + Tablet prompts teacher to provide support (observe students and provide feedback) for students with ADHD (non-EXP)(19)

Schoolbehwioml health prevention and early intervention system

3. Two-step Triage Procedure to support school health service st aff to provide digit al screening and additional intervention for highneed students (non-EXP) (20)

\begin{tabular}{|l|}
\multicolumn{1}{c|}{ C. effealth Targeting } \\
Pediatric CareSystem \\
(40\% based on EBPs)
\end{tabular}

FIGURE 2 | Pediatric eHealth strategies for families, schools, and pediatric care. EXP, Experimental design; Non-EXP, non-experimental design; Numbers included (e.g., (20)) were corresponding study number (not reference number) listed in Table 1 and Table 1S. EBls, Evidence-based interventions; EBPs, Evidence-based practices/guidelines. Box A summarizes purpose and eHealth strategies applies that target families. Box B summarizes purpose and eHealth strategies applied that target schools. Box C summarizes purpose and strategies applied that target pediatric cares.

Table 1 presents key findings for the 30 eHealth studies that were reviewed (from 32 articles), cataloged by eHealth intervention, country, targets, methods, design strategies, and impact or feasibility evidence (derived from Table 1S, the charting of review data in Supplemental file). Below, we summarize findings for the three areas of child behavioral eHealth literature. In each area of eHealth literature, we highlight target users, purpose of the eHealth design and strategies applied, and efficacy/effectiveness evidence.

\section{eHealth Targeting Families} Target Users

Most family-level eHealth interventions (for children 0-12 year olds) have targeted parent users (14 parenting-focused studies out of the total 17 family-focused eHealth studies), and fewer directly targeted child users (two child-focused out of 17 familyfocused studies) or a combination of parent and child users (1 out of 17). Parents and children were from either community or at-risk samples (e.g., children with behavioral challenges; families with multiple adversity indicators or high stress).
Purpose and eHealth Strategies Applied (Figure 2A)

For parent-targeted eHealth interventions, most interventions were developed to improve parents' child mental health knowledge and behavioral management skills/practices through multisession/module and multicomponent interventions (purposes). These types of interventions were usually adapted from existing EBIs (71\%). eHealth strategies applied include web-based parenting modules (included 4 to 12 interactive or structured parenting sessions), with (44, 45, 52-53, 54, 56, 57) or without $(42,47,48,50,55)$ additional e-communication or e-support for parents (e.g., phone coaching, e-reminders, feedback messaging, email, social media groups). There were also some studies focused on parent support interventions (including information and emotion support) and wellness promotion (purpose) for families of children with challenging behavior. For these support/wellness interventions, social media, and groupbased e-support strategies (e.g., Facebook, WhatsApp), with or without in-person sessions, were generally applied $(43,46,52)$.

For child-targeted eHealth interventions, one study focused on promoting child social skills and mental health (for 7-11 years 
TABLE 1 | Pediatric e-behavioral/mental health literature: study targets, methods, strategy, and impacts for the included articles.

\begin{tabular}{|c|c|c|c|c|c|c|c|c|c|}
\hline \multirow{2}{*}{$\begin{array}{l}\text { mHealth study/ } \\
\text { intervention }\end{array}$} & \multirow[t]{2}{*}{ Country } & \multirow{2}{*}{$\begin{array}{l}\text { Targets } \\
\begin{array}{l}\text { Parent, child, } \\
\text { provider }\end{array}\end{array}$} & \multirow{2}{*}{$\begin{array}{l}\text { Methods } \\
\begin{array}{l}\text { EXP, non-EXP, } \\
\text { review) }\end{array}\end{array}$} & \multicolumn{5}{|c|}{ mHealth strategies } & \multirow{2}{*}{$\begin{array}{c}\text { Impacts } \\
\text { Intervention effect }\end{array}$} \\
\hline & & & & $\begin{array}{l}\text { EBI/ } \\
\text { EBP (Y) }\end{array}$ & $\begin{array}{l}\text { Level } \\
\text { of TX } \\
(I, G, S)\end{array}$ & $\begin{array}{l}\text { Parenting digital } \\
\text { strategies }\end{array}$ & $\begin{array}{l}\text { Child digital } \\
\text { strategies }\end{array}$ & $\begin{array}{l}\text { Provider/system } \\
\text { digital strategies }\end{array}$ & \\
\hline \multicolumn{10}{|c|}{ Family-Level eHealth Strategies } \\
\hline $\begin{array}{l}\text { 1. Web-Based Parent } \\
\text { Management Training } \\
\text { for children with conduct } \\
\text { problems (42) }\end{array}$ & Sweden & $\begin{array}{l}\text { Parents of 3-12 } \\
y / 0\end{array}$ & EXP (RCT) & Y & 1 & $\begin{array}{l}\text { Web parenting training ( } 7 \\
\text { sessions) }\end{array}$ & & & Positive (on P\& C) \\
\hline $\begin{array}{l}\text { 2. Brief home visit parenting } \\
\text { intervention SafeCare } \\
+ \text { SafeCare-Facebook } \\
\text { parenting group for parents } \\
\text { at-risk for maltreatment (43) }\end{array}$ & US & Parents of $0-5 y / 0$ & $\begin{array}{l}\text { Non-Exp } \\
\text { (qualitative) }\end{array}$ & Y & I\&G & $\begin{array}{l}3 \text { weekly home visit } \\
\text { sessions + } 18 \text { Facebook } \\
\text { parenting network group }\end{array}$ & & & Positive (on P) \\
\hline $\begin{array}{l}\text { 3. Stepping Stone web- } \\
\text { based TX for promoting } \\
\text { parenting knowledge and } \\
\text { parent-child relationship } \\
\text { (44) }\end{array}$ & Korean & $\begin{array}{l}\text { Parents of } 11-16 \\
y / 0\end{array}$ & $\begin{array}{l}\text { EXP } \\
\text { (Quasi-Exp) }\end{array}$ & & 1 & $\begin{array}{l}\text { Web parenting training } \\
4 \text { weeks + weekly } \\
\text { telephone coaching }\end{array}$ & & & Positive (on P) \\
\hline $\begin{array}{l}\text { 4. Strongest Families Smart } \\
\text { Website (45) }\end{array}$ & Finland & Parents of 4 y/o & EXP (RCT) & Y & 1 & $\begin{array}{l}\text { Web parenting training } \\
\text { sessions (11 sessions) + } \\
\text { weekly phone coaching }\end{array}$ & & & Positive (on P \& C) \\
\hline $\begin{array}{l}\text { 5. Online Parent Social } \\
\text { Support for parents of } \\
\text { children with Special Health } \\
\text { Care Needs (CSHCN) (46) }\end{array}$ & US & $\begin{array}{l}\text { Parents of } \\
\text { CSHCN }\end{array}$ & $\begin{array}{l}\text { REVIEW (a } \\
\text { scoping review) }\end{array}$ & & $\mathrm{G}$ & $\begin{array}{l}\text { Parent-to-parent support } \\
\text { through digital media, } \\
\text { particularly social media } \\
\text { (Facebook) on emotional } \\
\text { and informational support }\end{array}$ & & & $\begin{array}{l}\text { Positive (on P, } \\
\text { especially for aged } \\
\text { 18-29 young } \\
\text { adults) }\end{array}$ \\
\hline $\begin{array}{l}\text { 6.ezPAREN, online } \\
\text { parenting training program } \\
(47,48)\end{array}$ & US & Parents of $2-5 \mathrm{y} / \mathrm{o}$ & $\begin{array}{l}\text { EXP (RCT } \\
\text { - Protocol) }\end{array}$ & Y & । & $\begin{array}{l}\text { Web/App-based self- } \\
\text { administered parenting (6 } \\
\text { modules) }\end{array}$ & & & $\begin{array}{l}\text { Effectiveness not } \\
\text { Yet Report, but } \\
\text { adherence and } \\
\text { user-engagement } \\
\text { were high. }\end{array}$ \\
\hline $\begin{array}{l}\text { 7. Triple P Online } \\
\text { Community (TPOC), an } \\
\text { online parenting program } \\
\text { with social media and } \\
\text { gaming features (49) }\end{array}$ & US & $\begin{array}{l}\text { Parents of } 2-12 \\
\text { y/o that are highly } \\
\text { vulnerable }\end{array}$ & $\begin{array}{l}\text { Non-EXP } \\
\text { (pre-post) }\end{array}$ & Y & I \& G & $\begin{array}{l}\text { Web parenting (eigth } \\
\text { sessions) + social media } \\
\text { group support + reward } \\
\text { system }\end{array}$ & & & Positive (on P) \\
\hline $\begin{array}{l}\text { 8. (a)(b) Triple P- Positive } \\
\text { Parenting Program- Online } \\
\text { Brief- TPOL Brief) }(50,51)\end{array}$ & Australia & $\begin{array}{l}\text { Parents of } 2-9 \\
\text { y/o with mild to } \\
\text { moderate conduct } \\
\text { problems }\end{array}$ & EXP (RCT) & Y & 1 & $\begin{array}{l}\text { Web parenting training } \\
(5 \text { modules) + Optional } \\
\text { technology assisted } \\
\text { communication tools } \\
\text { (e.g., remainder, summary } \\
\text { email) }\end{array}$ & & & Positive (on P \& C) \\
\hline
\end{tabular}




\begin{tabular}{|c|c|c|c|c|c|c|c|c|c|}
\hline \multirow{2}{*}{$\begin{array}{l}\text { mHealth Study/ } \\
\text { intervention }\end{array}$} & \multirow[t]{2}{*}{ Country } & \multirow{2}{*}{$\begin{array}{l}\text { Targets } \\
\begin{array}{l}\text { Parent, child, } \\
\text { provider }\end{array}\end{array}$} & \multirow{2}{*}{$\begin{array}{l}\text { Methods } \\
\begin{array}{l}\text { EXP, non-EXP, } \\
\text { review) }\end{array}\end{array}$} & \multicolumn{5}{|c|}{ mHealth Strategies } & \multirow{2}{*}{$\begin{array}{c}\text { Impacts } \\
\text { Intervention effect }\end{array}$} \\
\hline & & & & $\begin{array}{l}\text { EBI/ } \\
\text { EBP (Y) }\end{array}$ & $\begin{array}{l}\text { Level } \\
\text { of TX } \\
(\mathrm{I}, \mathrm{G}, \mathrm{S})\end{array}$ & $\begin{array}{l}\text { Parenting digital } \\
\text { strategies }\end{array}$ & $\begin{array}{l}\text { Child digital } \\
\text { strategies }\end{array}$ & $\begin{array}{l}\text { Provider/system } \\
\text { digital strategies }\end{array}$ & \\
\hline $\begin{array}{l}\text { 9. Parent-Wellness } \\
\text { WhatsApp Support Group } \\
\text { to promote mothers' } \\
\text { wellbeing of children with } \\
\text { autism spectrum disorder } \\
\text { (52) }\end{array}$ & $\begin{array}{l}\text { Saudi } \\
\text { Arabia }\end{array}$ & $\begin{array}{l}\text { Parents of } 26-78 \\
\text { month with } \\
\text { Autism spectrum } \\
\text { disorder }\end{array}$ & EXP (RCT) & & $I \& G$ & $\begin{array}{l}4 \text { WhatsApp-based } \\
\text { parent psychoeducation } \\
\& \text { support sessions + } \\
\text { 1-face-to-face session }\end{array}$ & & & Positive (on P) \\
\hline $\begin{array}{l}\text { 10. Cool Little Kids Online } \\
\text { Parenting training for } \\
\text { prevent child anxiety (53) }\end{array}$ & US & $\begin{array}{l}\text { Parents of 3-6 y/o } \\
\text { who are at-risk for } \\
\text { Anxiety }\end{array}$ & EXP (RCT) & Y & 1 & $\begin{array}{l}\text { Web parenting } \\
\text { training ( } 8 \text { modules) }+ \\
\text { Telephone consultation } \\
\text { by psychologist when } \\
\text { requested }\end{array}$ & & & Positive (on P \& C) \\
\hline $\begin{array}{l}\text { 11. COPING, a universal } \\
\text { web-based parenting } \\
\text { program to promote } \\
\text { positive parenting (54) }\end{array}$ & US & Parents of $3-8 \mathrm{y} / 0$ & $\begin{array}{l}\text { EXP (RCT } \\
\text { - Protocol) }\end{array}$ & Y & 1 & $\begin{array}{l}\text { Web parenting training } \\
\text { (10 sessions) }+ \\
\text { automated feedback }+ \\
\text { online praised message }+ \\
\text { text message reminders }\end{array}$ & & & Not Yet Report \\
\hline $\begin{array}{l}\text { 12. Universal internet- } \\
\text { based ParentWorks } \\
\text { parenting program (55) }\end{array}$ & Australia & $\begin{array}{l}\text { Parents of 2-16 } \\
\text { y/o }\end{array}$ & $\begin{array}{l}\text { EXP (RCT } \\
\text { - Protocol) }\end{array}$ & Y & । & $\begin{array}{l}\text { Online-based parenting } \\
\text { training ( } 8 \text { modules) }\end{array}$ & & & Not Yet Report \\
\hline $\begin{array}{l}\text { 13. Telephone-Supported } \\
\text { Triple P-Online Parenting } \\
\text { Program TPOL (TPOLe) } \\
\text { for parents with behavior } \\
\text { challenge children (56) }\end{array}$ & Australia & $\begin{array}{l}\text { Parents of } 1-8 \text { y/o } \\
\text { with disadvantage } \\
\text { or family difficulty }\end{array}$ & EXP (RCT) & Y & 1 & $\begin{array}{l}\text { Web parenting training } \\
\text { (TPOL) (8 sessions) } \\
+ \text { weekly telephone } \\
\text { consultation sessions for } \\
8 \text { weeks }\end{array}$ & & & Positive (on P\& C) \\
\hline $\begin{array}{l}\text { 14.Parenting Resilient } \\
\text { Kids (PRK), a web-based } \\
\text { parenting program for } \\
\text { child behavior problem } \\
\text { prevention (57) }\end{array}$ & Australia & $\begin{array}{l}\text { Parents of primary } \\
\text { school-aged } \\
\text { children }\end{array}$ & $\begin{array}{l}\text { EXP } \\
\text { (RCT- Protocol) }\end{array}$ & & 1 & $\begin{array}{l}\text { Web-based parenting } \\
\text { assessment + web- } \\
\text { tailored parent feedback } \\
\text { report + personalized } \\
\text { online modules (up to } 12 \\
\text { interactive modules) }\end{array}$ & & & Not Yet Report \\
\hline $\begin{array}{l}\text { 15. Adventures computer- } \\
\text { based game to improve } \\
\text { child social skills and } \\
\text { mental health (58) }\end{array}$ & US & $\begin{array}{l}7-11 \text { years old } \\
\text { with social skills } \\
\text { challenges }\end{array}$ & $\operatorname{EXP}(\mathrm{RCT})$ & Y & । & & $\begin{array}{l}9 \text { interactive online } \\
\text { adventure games } \\
\text { (include feedback, } \\
\text { prompts based on } \\
\text { performance) }\end{array}$ & & Positive (on C) \\
\hline $\begin{array}{l}\text { 16. Development of a } \\
\text { Digital Peer Support } \\
\text { Service (DPS) for children } \\
\text { coping challenges (59) }\end{array}$ & US & $\begin{array}{l}\text { 8-12 year-old } \\
\text { cancer survivor }\end{array}$ & $\begin{array}{l}\text { Non-EXP } \\
\text { (qualitative) }\end{array}$ & & $G$ & & $\begin{array}{l}\text { Personas-method } \\
\text { (an user-center } \\
\text { design method) was } \\
\text { used to co-design } \\
\text { DPS }\end{array}$ & & $\begin{array}{l}\text { DPS contents (3 } \\
\text { primary personas } \\
\text { for DPS) }\end{array}$ \\
\hline
\end{tabular}


intervention

\section{Parent, child,}

provider

EXP, non-EXP, EBI/

review)

EBP (Y)

\section{Parenting digital} (I, G, S)

17. BRAVE-ONLINE, webNew

7-15 year-old

Non-EXP (Pre-

based cognitive behavior

therapy (CBT) for childhood

anxiety (Primary focused on Zealand

children with

post follow-up

derate

anxiety after a

children, and secondary on

nature disaster

parents) (60)

School-Level eHealth Strategies

18. Web-based Learning

Management System

Brazil

(WBIE) for training teachers

on child mental health and

management (61)

19. iSelfControl, web-based

application to support

classroom behavioral

management for students

with ADHD (62)

20. Two-step Triage

Procedure for pediatric

Nether-

School health

Non-EXP

Non-EXP (13

US

Teachers and

9-11 years old

children with

ADHD

days follow-up

behavioral health preventive

old students

care in primary school (63)

feasibility study

\section{Pediatric Care/System eHealth Strategies}

21.Patient-Portal for

Parents in pediatric care:

feasibility \& usage (64)

22.Comprehensive

electronic previsit screener:

parent and provider

experience and impacts (65)

23. PEDStestOnline, a web-

based pediatric screening

\& clinical recommendation

(www.pedstest.com/online)

system (66)

$\begin{array}{llll}\text { US } & \begin{array}{l}\text { Parents and } \\ \text { Primary care } \\ \text { physicians (PCPs) } \\ \text { of 4-10 years old }\end{array} & \begin{array}{l}\text { Non-EXP } \\ \text { (mixed method) }\end{array} & \text { Y } \\ \text { US } & \begin{array}{l}\text { PCPs and parents } \\ \text { of 0-8 years-old }\end{array} & \begin{array}{l}\text { Non-EXP } \\ \text { (existing data } \\ \text { from 22 sites in } \\ \end{array} & \text { 20 states) }\end{array}$

6 web-education

videos $(9 \mathrm{hr})+$ internet

discussion forum + web

conference + support

text

Tablet prompts the

child to self-evaluate

and earn points for

adaptive behaviors

Tablet prompts the

teacher evaluate child

behaviors (record every

$30 \mathrm{~min}$ ) + view student

rating \& progress

+ view progress

\& compare with

teacher's rating

Parents of young

children

Non-EXP

(Cross-section

feasibility study)

child health account,

information, and record

management

Parent use of electronic

previsit screeners for child

behavioral health

Parent-portal for child

health screening (no

screening results) and

record management
A digital screening

carried out by SHS

assistants, and only

children in need of

follow-up were assessed

by the SHS doctors or

nurses

Patient-Portal integrated

with EHR system

Electronic Previsit

screening system for

parents and PCPs

Electronic screening

+ Parent-Portal with/

without integration with

EHR system
Intervention effect

Positive (on P\& C)

Positive for some $\mathrm{C}$

(for those with high inside)

Positive (on School preventive service)

Positive (on primary care service \& $P$ )

Positive (on primary care service, $P$ and PCPs)

Impact evidence not reported (Report

only patterns of

utilization and

implementation) 


\begin{tabular}{|c|c|c|c|c|c|c|c|c|c|}
\hline \multirow{2}{*}{$\begin{array}{l}\text { mHealth Study/ } \\
\text { intervention }\end{array}$} & \multirow[t]{2}{*}{ Country } & \multirow{2}{*}{$\begin{array}{l}\text { Targets } \\
\begin{array}{l}\text { Parent, child, } \\
\text { provider }\end{array}\end{array}$} & \multirow{2}{*}{$\begin{array}{l}\text { Methods } \\
\begin{array}{l}\text { EXP, non-EXP, } \\
\text { review) }\end{array}\end{array}$} & \multicolumn{5}{|c|}{ mHealth Strategies } & \multirow{2}{*}{$\begin{array}{c}\text { Impacts } \\
\text { Intervention effect }\end{array}$} \\
\hline & & & & $\begin{array}{l}\text { EBI/ } \\
\text { EBP (Y) }\end{array}$ & $\begin{array}{l}\text { Level } \\
\text { of TX } \\
(\mathrm{I}, \mathrm{G}, \mathrm{S})\end{array}$ & $\begin{array}{l}\text { Parenting digital } \\
\text { strategies }\end{array}$ & $\begin{array}{l}\text { Child digital } \\
\text { strategies }\end{array}$ & $\begin{array}{l}\text { Provider/system } \\
\text { digital strategies }\end{array}$ & \\
\hline $\begin{array}{l}\text { 24. Public available Educational } \\
\text { Videos for managing children's } \\
\text { pain and anxiety generated by } \\
\text { needles (30) }\end{array}$ & Global & $\begin{array}{l}\text { Parents and PCPs } \\
\text { of infants and } \\
\text { toddlers }\end{array}$ & $\begin{array}{l}\text { REVIEW (a } \\
\text { scoping review) }\end{array}$ & & I & $\begin{array}{l}\text { Web-based behavioral } \\
\text { management strategy } \\
\text { videos for parents or } \\
\text { PCPs }\end{array}$ & $\begin{array}{l}\text { child pain/anxiety } \\
\text { management } \\
\text { strategy videos }\end{array}$ & $\begin{array}{l}25 \text { Public Educational } \\
\text { Videos from YouTube \& } \\
\text { Google search }\end{array}$ & $\begin{array}{l}\text { No impact evidence } \\
\text { reported }\end{array}$ \\
\hline $\begin{array}{l}\text { 25. eRedBook, a digitized } \\
\text { version of EHRs in UK: } \\
\text { Implementer and user } \\
\text { experience and barriers (67) }\end{array}$ & UK & $\begin{array}{l}\text { School health staff } \\
\text { and parents of } \\
\text { young children }\end{array}$ & $\begin{array}{l}\text { Non-EXP } \\
\text { (Qualitative } \\
\text { study) }\end{array}$ & Y & I\&S & $\begin{array}{l}\text { Parent-portal for health } \\
\text { management (enrolled by } \\
\text { public health nurses and } \\
\text { health visitors) }\end{array}$ & & $\begin{array}{l}\text { Enrolling parents to use } \\
\text { EHR-Integrated Parent- } \\
\text { Portal system }\end{array}$ & $\begin{array}{l}\text { Negative (Many } \\
\text { enrollment barriers; } \\
\text { e.g., safety, } \\
\text { complexity, literacy, } \\
\text { WiFi availability) }\end{array}$ \\
\hline $\begin{array}{l}\text { 26. An EHR-based tool } \\
\text { with names, photos, and } \\
\text { definitions of treatment } \\
\text { team members to increase } \\
\text { parents' accuracy in } \\
\text { identifying care team (68) }\end{array}$ & US & $\begin{array}{l}\text { Parents in } \\
\text { pediatric care }\end{array}$ & $\begin{array}{l}\text { Non-EXP (pre- } \\
\text { post survey) }\end{array}$ & & I\&S & $\begin{array}{l}\text { Parent use of integrated } \\
\text { EHR functions to identify } \\
\text { care team members \& to } \\
\text { build trust }\end{array}$ & & $\begin{array}{l}\text { An EHR function to } \\
\text { improve parent-care } \\
\text { team communication }\end{array}$ & Positive (on P) \\
\hline $\begin{array}{l}\text { 27. eHand-over Tool, an } \\
\text { EHR-integrated medical } \\
\text { handover tool to improve } \\
\text { medical handover between } \\
\text { doctors (69) }\end{array}$ & Australia & $\begin{array}{l}\text { Pediatric } \\
\text { Providers }\end{array}$ & $\begin{array}{l}\text { Non-EXP } \\
\text { (cross-sectional } \\
\text { survey) }\end{array}$ & & $S$ & & & $\begin{array}{l}\text { An EHR-integrated } \\
\text { tool to standardize } \\
\text { and improve both the } \\
\text { standard and efficiency/ } \\
\text { efficacy }\end{array}$ & $\begin{array}{l}\text { Positive (on } \\
\text { pediatric care, } \\
\text { Dr. satisfaction \& } \\
\text { workflow) }\end{array}$ \\
\hline $\begin{array}{l}\text { 28.EHR-integrated } \\
\text { strategies to increase } \\
\text { discharge communication } \\
\text { in multidisciplinary team \& } \\
\text { Impacts (70) }\end{array}$ & US & $\begin{array}{l}\text { Pediatric PCPs } \\
\text { \& hospitals } \\
\text { physicians }\end{array}$ & $\begin{array}{l}\text { Non-EXP } \\
\text { (Qualitative } \\
\text { study) }\end{array}$ & & $S$ & & & $\begin{array}{l}4 \text { strategies to improve } \\
\text { communication: } \\
\text { Standardize process; } \\
\text { Make it Easy; Eliminate } \\
\text { waste; \& Incentivize }\end{array}$ & $\begin{array}{l}\text { Positive (on } \\
\text { Pediatric and } \\
\text { Primary care } \\
\text { service) }\end{array}$ \\
\hline $\begin{array}{l}\text { 29. SHARE intervention, } \\
\text { a multicomponent } \\
\text { distance-learning/quality } \\
\text { improvement program } \\
\text { to improve PCPs' use of } \\
\text { ADHD rating scale (71) }\end{array}$ & US & $\begin{array}{l}\text { Pediatric care } \\
\text { practices and } \\
\text { PCPs }\end{array}$ & $\operatorname{EXP}(\mathrm{RCT})$ & & $S$ & & & $\begin{array}{l}\text { SHARE includes: } \\
\text { Web-based education; } \\
\text { Collaboratively } \\
\text { consultation with ADHD } \\
\text { experts; and Performance } \\
\text { feedback reports/calls }\end{array}$ & $\begin{array}{l}\text { Positive (on PCPs } \\
\text { and Primary care } \\
\text { service) }\end{array}$ \\
\hline $\begin{array}{l}\text { 30.Digital Education } \\
\text { Program Development to } \\
\text { Train nurse in caring for } \\
\text { children with self-harm } \\
\text { injuries (72) }\end{array}$ & US & $\begin{array}{l}\text { Nurses in } \\
\text { pediatric care }\end{array}$ & $\begin{array}{l}\text { Non-EXP (a } \\
\text { participatory } \\
\text { approach) }\end{array}$ & & $S$ & & & $\begin{array}{l}\text { e-Learning program for } \\
\text { nurse that is sensitive } \\
\text { to nurses' and care } \\
\text { recipients' needs }\end{array}$ & $\begin{array}{l}\text { No impact } \\
\text { evidence reported } \\
\text { (Report digital } \\
\text { tool development } \\
\text { process) }\end{array}$ \\
\hline Total & $\begin{array}{l}16 \text { US; } \\
1 \text { LMIC; } \\
13 \\
\text { Others }\end{array}$ & $\begin{array}{l}17 \text { Family; } 3 \\
\text { School; } 10 \\
\text { Pediatric }\end{array}$ & $\begin{array}{l}14 \text { EXP; } 14 \\
\text { Non-EXP; } 2 \\
\text { Reviews }\end{array}$ & $17 Y$ & $\begin{array}{l}14 \text { I; } \\
2 \text { G; } 3 \\
\text { I+G; } 4 \text { S; } \\
7 \text { I+S }\end{array}$ & $\begin{array}{l}21 \text { Parents (14 } \\
\text { parenting training; } \\
2 \text { parent Support- } \\
\text { Groups; } 5 \text { EHR-Portal) }\end{array}$ & $\begin{array}{l}5 \text { Children ( } 2 \\
\text { educational video; } \\
1 \text { game, } 1 \text { behavior } \\
\text { regulation, } 1 \text { peer } \\
\text { support) }\end{array}$ & $\begin{array}{l}15 \text { System ( } 3 \text { school } \\
\text { system; } 10 \text { pediatric } \\
\text { system) }\end{array}$ & $\begin{array}{l}23 \text { Impact } \\
\text { Reported (21 } \\
\text { positive; } 2 \\
\text { Negative or } \\
\text { uncertain) }\end{array}$ \\
\hline
\end{tabular}

TX, Intervention. Level of TX; I, individual family/child-focused; G, family/child group-focused; S, system-focused (school or pediatric care system). EBI/EBP, Evidence based intervention (defined as intervention adapted from EBI that used nondigital approach or digital-based EBI)/Evidence-based guideline (defined as evidence-based practice guideline recommended by professional pediatric society); Y, Yes.

Exp, Experimental evaluation (e.g., RCT, experimental comparison, quasi-experimental). y/o, years old. Intervention Effect: P, Parents; C, Child; T, Teacher; PCP, Primary care physicians; 
old) through use of online gaming sessions (including 9 game sessions, with feedback and prompts based on performance). The game components included game goals, rules, game mechanics, and procedures to generalize or transfer game learnings to daily life (58). Another study focused on the development of digital peer support tools by first focusing on content development, using a user-centered and participatory method to design personas (59). Yet other study targeted parent and child simultaneously; this multiuser and more intense treatment intervention focused on children with mild to moderate anxiety with an adaptation of cognitive-behavioral therapy (CBT) to a digital approach (with e-training modules and digital communication support) (60).

\section{Efficacy/Effectiveness Evidence (Table 1 and Table 1S)}

Among the reviewed parent-focused eHealth studies that had outcome data (11 studies), 7 were evaluated using experimental designs (70\%). All 11 eHealth parent-focused interventions reported positive impacts on parenting, and six interventions also reported positive impacts on child mental health (based on short term or less than one-year follow-up period). Among the reviewed child-targeted eHealth studies that had child outcome data (2 studies), one used an experimental design, and all child studies (with 9-10 eHealth sessions) showed positive impacts on child mental health $(58,59)$.

\section{eHealth Targeting School Mental Health Services \\ Target Users}

Among the identified school-focused eHealth literature that promotes young children's mental health ( 3 studies), interventions targeted teachers ( $1 / 3$ studies), school health personnel $(1 / 3$ studies), or students who had behavioral or mental health challenges (with teachers' involvement; 1/3).

\section{Purpose and eHealth Strategies Applied (Figure 2B)}

eHealth in school contexts has been applied to strengthen school mental health resources or improve school mental health care. For the eHealth that targeted teachers, the intervention was designed to train teachers on child mental health and classroom behavioral management (purpose). It used a web-based learning management system (WBIE) approach, including web-education videos (6 modules), online discussion forum, web conference and support text messaging, to support teachers' child mental health education and practices (61). This study was conducted in an LMIC (Brazil). For the eHealth that targeted school health personnel, the intervention was designed to entail a two-step triage approach to support school behavioral health services. In Netherlands, community-based school-health professionals (e.g., physicians, nurse, and health assistants) visit schools a few times a year for routine child health and behavioral health assessments. For students identified with problems or needs, additional services are provided. The eHealth two-step triage strategy was designed to provide preassessments (a digital screening questionnaire) to primary school students by trained school health assistants, as well as a built-in follow-up decision support function that allows the school health team to be notified to follow need-identified students (63). For the eHealth that targeted students with behavioral challenges, the intervention was design to improve ADHD students' self-regulation in classrooms by involving target students and their teachers in the behavioral observation-feedback loop (purpose). The eHealth strategy was to use a digital tool (iPad-based) to: (i) prompt students to self-evaluate their own adaptive behaviors (e.g., following instructions/rules, staying on task), (ii) earn rewards (points added or subtracted from their account); and (iii) view teacher feedback and compare self-ratings with teacher ratings of their behaviors (62).

\section{Efficacy/Effectiveness Evidence (Table 1 and Table 1S)}

Two of the school-focused eHealth interventions reported positive impacts on teachers or school health services $(61,63)$. Only the study conducted in the LMIC (for teacher education about child mental health and behavioral management) was evaluated using an experimental design (61). The ADHD digitaltool intervention only benefited students with high insight (62), indicating the importance of considering user characteristics in eHealth design and implementation.

\section{eHealth Targeting Pediatric Care Settings Target Users}

Most eHealth in pediatric care focused on pediatric professionals, adults who care for children, or users of EHR systems (i.e., parents, pediatric care providers/PCPs, pediatric care team).

\section{Purpose and eHealth Strategies Applied (Figure 2C)}

For eHealth interventions that targeted pediatric professionals or adults who care for children, the interventions were usually designed for education purposes. For example, e-learning strategies and web-education models/videos might be applied to educate/train adults or professionals to screen or care for children with mental health related problems (e.g., ADHD, self-harm, anxiety) $(30,71,72)$. The e-learning strategy might be combined with other e-collaborative/consultation or performance feedback communication functions (e.g., report or call) to provide additional support (70).

For eHealth interventions that targeted EHR users, two groups of studies were identified. One group of studies focused on promoting patient-portal usage to improve preventive mental health screening, record management for patients/parents $(64,67)$, and/or decision support for providers (e.g., integrate screening/decision notifications for providing additional services) $(65,66)$. For screening and patient record management, web-based or EHR-based previsit screening and e-account management strategies have been applied. These e-strategies were implemented through navigator assistance or self-serve account setup. For provider decision support, integrated screening/EHR strategies (e.g., with automatic scoring, alter-notification/prompt for action when at-risk case identified, and automated decisions that is built into the clinical workflow) have been applied.

The other group of EHR studies focused on promoting communication and information sharing among the pediatric care team. This could be either improving communication 
between patient and care team (68) or improving communications (standard, procedure, information format) among medical care team members $(69,70)$. eHealth strategies such as e-demonstration and integration of standardized activities/ practice guidelines/forms/prompts with EHR processes have been applied.

\section{Efficacy/Effectiveness Evidence (Table 1 and Table 1S)} Among the three reviewed e-learning studies (targeting pediatric professionals or adults who care for children), only one study evaluated the outcome using an RCT design (70). This study found that distance-learning that integrated web-based education, collaborative consultation, and performance feedback for PCPs on child mental health screening could increase PCPs' use of behavioral screening tools in pediatric care.

Among the EHR studies that focused on promoting patientportal use, most showed positive evidence (2 of 3 studies), using a nonexperimental design. Parents showed improvement in use of EHR-portal or screening tools and positive pediatric care experience $(64,65)$. However, one study showed negative findings and challenges while implementing a patient-portal due to technology issues (e.g., web access issues, poor technology literacy in users) (67).

Among the EHR studies that focused on promoting communication and information sharing, all studies (three of three) reported positive impacts and user experience (e.g., increase use of e-communication tools/procedures, improvement in communication, care workflow) (68-70). However, none of these were evaluated using experimental designs or had reported impacts on child mental health outcomes.

\section{eHealth Design Strategies for User Engagement and Usability}

To draw lessons learned from the existing eHealth research on design strategies for engaging users in eHealth interventions (or design strategies that maximize products' usability, accessibility, and target users' needs) to inform future eHealth development in LMIC contexts, we synthesize findings from studies that discussed or provided evidence related to user engagement/ user-centered strategies, usage patterns, level of engagement, and satisfaction of eHealth interventions. Twenty-one studies out of 32 studies that we reviewed provided these user engagement results and discussion; therefore, these articles were used for research synthesis.

Based on the available articles, we grouped user-engagement design strategies and usability evidence/lessons into four areas of eHealth applications. These include user-engagement strategies and usability lessons related to: i) e-parenting intervention design, ii) health worker eLearning/e-training design (i.e., eLearning strategies for pediatric providers, school health staff), iii) designing integrated e-screening and e-decision-support tools in primary care, and iv) designing workflow integrated e-communication/collaboration tools. We summarize findings in the sections below. Tables 2A, B also summarize key lessons from our analysis. User-engagement strategies marked with ${ }^{* *}$ in Table 2 were those that we believe are relevant to LMIC contexts.

\section{User-Engagement Strategies and Usability in e-Parenting Intervention Design}

In engaging parents to use e-parenting interventions (i.e., e-parenting in individual or group format), several strategies related to parent recruitment, technology literacy, privacy/safety, motivation to use e-parenting technology, and engagement in learning were identified as critical. For example, recruiting parents from traditional face-to-face practices would be a better recruitment strategy (e.g., with better retention rate) than recruiting parents from social media given personal contacts promoting more social bonds and therapeutic alliance relationship (44). To address technology literacy challenges in e-parenting technology use, including an introduction session to help parents sign-up/set up an account and learn about e-tool functionality can be a helpful strategy (43). To promote parents' participation frequency in use of eHealth tools/modules, including incentive strategies (e.g., use raffle tickets, win prizes, achieving badges, use of social network, promote online communities) and additional communication strategies (e.g., contact with the interventionists, use group messages, regular reminders, instant chat, technical support) are critical for improving effectiveness and usability $(43,49)$. To support parents learning and behavioral changes, eLearning with some forms of consultation or learning support (e.g., from implementers or other experienced parent-peers) and integrating parents' preference of learning styles/activities (e.g., working in groups with similar aged children, geographic locations) can bolster parenting behavioral changes or support $(49,53,56)$.

\section{Usability}

Eight out of 14 parent-focused interventions reported eHealth usability. Three key lessons were gleaned from the findings: (1) frequency of eHealth usage would be higher when the accessibility of the digital devices is high and easy to use (in format that matches with target users' life style), and when resources/contents were relevant and more interactive (43) (49); (2) full completion rate for all modules/session increases $(35 \%-$ $66 \%$ ) when the eHealth interventions are well designed (using short modules [15-30 minutes/per module], includes five or more sessions, and includes multiple communication supports (e.g., 20 minutes/per contact) $(42,47,49-50,51,56,60)$; and (3) when social network/e-community and optional technologyassisted communication tools were included, the likelihood to complete a minimum dose of intervention and user satisfaction can generally increase $(50,51,56)$.

\section{User-Engagement Strategies and Usability in Health Worker eLearning/e-Training Design}

In training health workers (i.e., school staff, pediatric providers), one important lesson gained was that eLearning using web-based and offline video approaches were found to be equally effective in training. This is especially relevant to LMIC contexts, given challenges in internet access in many regions. Furthermore, interactive web-based education that also included other learning support (e.g., a discussion forum, opportunities to interact with consultants/child psychiatrists, web-conferencing, performance 
TABLE 2 | eHealth user engagement strategies, usage patterns and acceptability evidence.

(a) eHealth user engagement strategies (** Lessons also relevant to low- and middle-income countries (LMICs))

eHealth in Contexts User-Engagement Strategies

eHealth in Parenting Intervention Context - Parent recruitment via social media vs. general practices ${ }^{\star *}$ : Parents recruited from general practices tend to stay on to the program for longer time than parents recruited from social media (given established social bonds or therapeutic alliance relationship) (44)

- Strategies for addressing Technology literacy**: For families with low technology literacy or not use technologies on regularbasses, including one introduction session in the beginning of parenting program to help sign-up a private group account, and demonstrate online tool functionality can be helpful (43)

- Privacy/Safety strategies**: Parents prefer respectful communication and information sharing. Contents that they or other parents share should be careful chosen and appropriate. Parents felt comfortable sharing parenting information with an anonymous group (43)

- Motivators or incentive strategies to promote e-parenting technology use**: (i) promoting relationship tides between parents and the online communities/social networks $(44,56)$; (ii) including a social network group in e-parenting intervention (social network as a sharing community); (iii) inclusion of Facebook "events", (iv) including incentive approaches (e.g., raffle tickets, win prizes, gaming approach/achieving badges) to promote participation motivation, (v) having more contact with interventionist through social media channels (group messages, regular reminders, instant charts) $(43,49)$

- Learning Engagement for Parents ${ }^{\star *}$ : i) parents prefer working in group that have more similarities between parents themselves and other parents (e.g., similar-aged children, geographic locations); ii) parents commented that it would be helpful to see more examples of the skills that other parents are participating (from interventionist or other parents) (43); iii) web-based eLearning with some forms of consultation or learning support (from earlier cohort of parents or implementers) can promote parents' skill learning and total number of session completion rate $(49,53,56)$

eLearning in Provider/System Context (for PCPs or School Teachers)

E-Screening \& E-Service Decision Support (in School Health Service or Primary Care Context)

E-Communication in Care Team Collaboration Context
- Web vs. offline eLearning strategies ${ }^{\star *}$ : For staff e-training, both web-based interactive education (WBIE; including a discussion forum to interact with consultants, and a web conference with a child psychiatrist) and the video-based education (TVBE; including receiving text, but not in-person connection) can be effective eLearning approaches (e.g., both types of eLearning had more nonstigmatized concepts than the control). However, the WBIE interactive approach was more effective than the TVBE noninteractive approach (e.g., in knowledge gain, fewer stigmatized concepts/opinion). Results suggesting adding a discussion forum and web conferencing can have more knowledge gain, but not on changing attitudes (61).

- A multicomponent eLearning strategy**: a three-component distance-learning can be effective, which includes web-based education [3 15-min modules], collaboratively consultation with child mental health experts, and performance feedback report/calls (71)

- Co-developing training/educational materials ${ }^{\star *}$ is an important way for designing educational resources that included a strong patient voice, meet nurse learning needs, and ensures the content is relevant, appropriate and sensitive to both the recipient of care and those responsible for its delivery (72)

- Integration of two-step triage procedure is a good and acceptable way to set up preventive behavioral health care in schools ${ }^{\star *}$. The two-step triage includes a digital screening conducted by health assistants (task-shifting to community health workers) and a referral or additional service conducted by health professionals for those screened positive (task-sharing) (63)

- Patient portal enrollment and engagement strategies (in primary care) ${ }^{\star \star}$ : i) Use a navigator to demonstrate the patient portal to parents can increase the sign-up rate and address parent's technology literacy barrier (64); ii) Provide computers in the waitingroom and using waiting-room attendant (e.g., gap year students or retirees paid close to minimum wage, who can help interview families with limited literacy or can be charged with entertaining children, modeling appropriate adult-child interaction, implementing Reach and Read) that address family technology access and technology literacy gaps to improve enrollment and portal use (56\%) in comparing to a reminder option (which give parents an appointment reminder card, including information on how to log-in to PEDS online and request to complete screens before the next scheduled visit) (44\%) (66);

- Web- vs. On-site kiosks approach for setting up Patient Portal: Many parents who sign up for patient portal also wanted to have the access of patient portal (for their child) via on-site kiosks (64)

- Integration of Previsit screening with EHR Decision Support: Parents like this approach when: i) the digital approach is easy to use; ii) the screening highlighted the areas of concern that the doctor needed to touch on/discuss during the well-child care visit; iii) the screening questions relevant to their needs/questions (which remind them areas of concerns they may discuss with providers or areas of child health issues that they have not thought of; iii) completing the screener in advance that improve visit efficiency. Providers like the approach when ${ }^{* *}$ : i) reducing the workload; ii) a summary report was provided to discuss with parents; iii) comprehensive screening questions were used (e.g., inclusion of mental health and other nonphysical health issues on the screener) that eliminating the need for the PCP to take the time to assess these issues $(65,67)$

- e-Strategies for trust-relationship between patients and care team members ${ }^{\star \star}$ : including names, photos, and definitions of treatment team members (e.g., role) can promote parent ability to correctly identify the care team, trust relationship, and care satisfaction (68)

- Four strategies to enhance pediatric care team communication**: 1) development of standard process for e-Handover; 2) reduction of overproduction and defects by "Making it Easy to Follow the Standard" and by providing resident/care team education; 3) eliminating waste in wait and search times (by improve use of EHR for communication, e.g., supplying care team members with providers' phone number and prefer methods of contact); and 4) aligning the incentive with those performing the work (70) 
TABLE 2 | Continued

(b) eHealth Usage Patterns and Acceptability Evidence

\section{eHealth in Contexts \\ eHealth Usage Patterns and Acceptability}

eHealth in Parenting Interventions

- Safe-Care Facebook Social Support group: Use Frequency: Families with computers at home were more likely to check Facebook regularly (e.g., 3 times weekly) than families that relies on public facilities (e.g., computer in library; e.g., participate in some weeks). Parents commented on the content that they enjoyed viewing (of others' postings) within the group including parenting resources, links to websites, and supportive comments to and from other parents (43)

- Web-PMT (7 self-paced parenting sessions): 66\% completed all 7 sessions, 22\% completed 3-6 sessions, 16\% completed fewer than 3 sessions. 69\% families with two parents participated together (42)

- ezPARENT (6-sessoin Parenting program self-administrated modules) (in the US): On average, parents spent 37.2 min per module (SD = 22.2); the mean number of program visits was 13.6 (SD = 8.6; range 2-49). Average length of time per visit was 14.1 $\min (\mathrm{SD}=17.1)$. Participants completed on average $82 \%$ of the modules (out of 6 total modules) (47).

- Triple P Online Community (TPOC) (8 online modules + social media + incentive in the US): online modules were access through numerous channel, such as agency computer lab (70\%; home computer (54\%), cell/smart phone (51\%), work or school computer (33\%), iPad or tablet (31\%), friends' computer (21\%), free WiFi (restaurant, 20\%), public library computer (16\%). The complete rate for the entire 8-module program was 36-51\% (higher rate when smartphone is available, in later cohort/with support from earlier cohort)(71)

- Triple P Brief (TPOL Brief)-(Self-directed 5 modules, with optional technology assisted communication tools (e.g., text prompts reminder, send module summary via email): $62 \%$ completed at least the recommended minimum dose (introductory module + one exemplar module), $53 \%$ completed 3 or more, $45 \%$ completed 4 or more, $40 \%$ completed all 5, 13\% completed introductory only, $25 \%$ did not completed any. Average module completion time was around 2 hours for the introduction and 45 min for the exemplar modules which is longer than expected, indicating that parents were explored optional extra material. $88 \%$ rate the program as good, and $77 \%$ were at least satisfied with the program. Parents with high disagreement over parenting were less likely to complete minimum dose of intervention $(50,51)$

- TPOL-with weekly Telephone support (8 weekly online module + telephone consultation): parents in the TPOLe condition completed significantly more modules and higher satisfaction than directed TPOL ( $\mathrm{M}=5.62$ and $3.25 ; 47 \%$ vs. $23 \%$ completed all 8 modules). Mean module completion time was 63 minutes. TPOLe group participated in $4.36(S D=2.53)$ clinical telephone support session on average. Average call duration was 24 minutes $(S D=8)$. There was a significant correlation between the number of telephone consultations and number of online modules completed (56)

- Online-Cool Little Kids parenting program (8 online modules + telephone consultation when requested): Online program use was lower than the high attendance rates generally observed for the group parenting program when delivered through a university research clinic. Only $1 / 3$ of parents attended most sessions (53)

- BRAVE-ONLINE (eCBT; 10 online sessions for children, and 5-6 online sessions for parents): On average, by 6-month follow-up, children and adolescents had completed 5.9 of 10 sessions (SD = 2.9) and parents had completed 4.56/6 (SD = 1.7)(child parent program) and $2.95 / 5$ (SD = 1.9) sessions (adolescent parent program); $35 \%$ and $41 \%$ had completed all their sessions at the time of the follow-up (60)

eLearning for Providers or Teachers

- For school-based e-Training (for promoting teacher mental health in LMICs), the attrition rate was high across all group: $31 \%$ for WBIE and 52\% for TVBE (might be due to high rates of teacher absenteeism and time unavailability due to high workloads). If participated, the impact was positive after receiving training (61)

- For Pediatric care-based eLearning: 79\% completed all 3 educational presentations. There were on average two phone, email, or in-person consultations with ADHD experts per month. 57\% clinicians participated in at least one performance feedback call. Use of Care Assistant were more frequent in intervention (36\% use at least 5 times, and 19\% use at least 10 times) than in Control (31\% use at least 5 time, and $15 \%$ use at least 10 times). Intervention clinicians who participated in at least one performance feedback call were more likely to send out parent rating scales than intervention clinicians who did not participate (relative difference of 14.2 percentage points (71).

(Continued)

feedback calls/report) can further promote health workers' skill learning, behavioral changes, and session completion rate (61, 71). For content design, codeveloping E-educational materials/ content between health workers and targeted children/families can better meet health workers' needs, and ensure the content is relevant, appropriate and sensitive to both the recipients of care and those responsible for its delivery (72).

\section{Usability}

E-learning in LMICs has been found to be more challenging than e-Learning in developed countries, with a lower reach/usage rate in LMICs (i.e., $48 \%-70 \%$ reach in LMIC-Brazil vs. $79 \%$ in developed countries). Reasons for low reach include high rates of staff absenteeism, workloads, and time constraints, all of which indicate the importance of addressing contextual barriers in
eLearning designs $(61,71)$. When performance feedback sessions were provided (to offer in-person consultation/support to answer trainees' questions about e-Learning content or to promote use of skill after eLearning), just over half (57\%) of trainees participated in at least one feedback call. Those who used feedback calls were more likely to adopt the skills learned in eLearning (71). Findings suggest the importance of standardizing feedback sessions as part of the eLearning delivery models.

\section{User-Engagement Strategies and Usability in Designing Integrated e-Screening and Electronic- Service-Decision-Support Tools for Primary Care} To promote provider and parent use of an integrated e-screening and decision support tool (with triage function), userengagement strategies targeting parent users and care provider 
TABLE 2 | Continued

(b) eHealth Usage Patterns and Acceptability Evidence

\section{eHealth in Contexts \\ eHealth Usage Patterns and Acceptability}

E-Screening \& E-Service Decision Support (in School Health Service or Primary Care Context)

- Schools using the 2-step triage procedure to set up preventive child behavioral health service, they provide more accessible service to students (measured by increasing utilization of/contacts \# with school health service professionals than the schools not using 2-step triage. This approach is also perceive as an more appropriate approach to support children with special needs (63)

- Implementation of Pediatric Screening by Providers (use of PEDStestOnline (www.pedstest.com/online): PEDS (evaluation of developmental status) was most commonly used (100\%), followed by PEDS : DM (evaluate developmental milestones) (41\%) and M-CHAT (Autism screening)(21\%). Use of the M-CHAT spiked around 18months of age and remained high in the months surrounding 24 months of age. The screening use decreased ager 3 years of age (66).

- Staffing for Implementing Pediatric-Screening: Receptionists/medical technician stations were often served as the point for dissemination clipboards/screening measures (at check-in). Next, skilled nurses often in charged with entered parents' responses into PEDS Online, offering an interview if forms were incompleted (clarified parents' comments and answers to items)or if evidence of limited literacy was present (66)

- Patient Portal Usage (for screening and health record management): In the US, less than 50\% clinics implemented webportal. For the clinics that implemented, \% family used portal varied (range of family reached was 35\%-100\%), and only $10 \%$ use previsit screening. English speaking parents were more likely to use the online portal and screening thank non-English speaking families, but no difference in presence or absence of porta use due to parents' level of education or poverty. Among the users, about $70 \%$ of parents reported that they planned to use the patient portal again for their child (after sign-up). Since activation, median use was 0.8 times per month. A two-year tracking among disadvantaged populations found $81 \%$ of patients who activated their accounts accessed the portal twice or more (64)

- Parent use of previsit screening tools: Parents indicated high acceptability of the screeners. Nearly $90 \%$ trusted the security of the screener, and $87 \%$ thoughts their answers would be confidential; $92 \%$ thought the screener was a good way to ask routine questions, 95\% were comfortable with the mental health questions, and 89\% thought the screener helped with sharing of concerns (65)

E-Communication in Care Team Collaboration

- After implementation of EHR-Care team introduction system, parents showed improved rate for correctly identifying care team physicians (71\% s 28\%). Most parents (79\%) and care-team members (87\%) also reported that subjects' ability to identify care team members impacted their satisfaction and trust relationship (68)

- Communication compliance and process significantly improve after implementation of e-handover communication Tools: 15 out of 19 services have improved communication; satisfaction increase from 17\% to 678\%-7\%) (69); 81\% of PCPs were followed and confirm that e-handover communication was received (70); the Tools significantly simplify the care process (measured by $68 \%$ of PCP reported significantly reducing paper work time, and reduction of redundant data from 52\%) (69)

users (including physicians, coordinated implementers) need to be considered. Efficient strategies that have been identified to promote patient-portal use/enrollment and use of previsit screening for their children include: use of navigators/waitingroom attendants (to demonstrate, assist sign-up, support parents who have low literacy), providing computers/on-site kiosks in waiting rooms, using reminder cards (with specific requests, instructions and information for using e-screening), keeping digital functions easy to use, making screening questions relevant to parents' needs, and summarizing areas for further attention (or highlighting areas for further discussion with health providers) (64-67). To promote provider uptake and use of integrated e-screening and decision support tools in pediatric care (for behavioral health promotion), providing training on the digital tool use (with clear explanation of functions and clinical workflow processes) and providing tools that have multiple benefits in meeting providers' needs (e.g., reducing workload, improving clinical efficiency, a summary report to discuss with parents, including comprehensive screening questions that eliminating the need for providers to take the time to assess) are critical to consider in design and delivery models $(63,65,67)$.

\section{Usability}

Use of a parent portal for routine pediatric care is relatively new in pediatric behavioral health care settings in the US. Results of the
PEDStestOnline study (that included 79 providers across 20 states, with data from 20,941 children ages birth to 8 years) indicated that $30 \%$ of sites implemented a web portal, and the rate of family enrollment varied by providers (reach 35\%-100\% families). Additionally, only $10 \%$ of eligible families used a previsit screening. The rate of uptake could depend on enrollment approaches. Englishspeaking parents were more likely to use the screening and online portal than non-English speaking parents, but there were no differences in portal use by parents' level of education or poverty (66). For clinics that provided computers in the waiting room, the uptake for portal use was higher (56\%) than the clinics that used the appointment reminder approach of enrollment (44\%). For those parents enrolled, about 70\% reported that they planned to use the portal again for their child, and $81 \%$ continued accessing the portal two years after initial enrollment (66). Parents also reported high acceptability and satisfaction (e.g., about $90 \%$ trusted the security of the screener, $92 \%$ thought the screener was a good way to ask routine questions, 95\% were comfortable with the mental health questions, and $89 \%$ thought the screener helped with sharing of concerns) (65).

\section{User-Engagement Strategies and Usability in Designing Workflow Integrated e-Communication/ Collaboration Tools}

Applying collaborative and team-based care models to integrate behavioral health service in routine primary care is 
a recommended clinical practice guideline for pediatric care, especially for addressing the needs of special or high-risk pediatric populations (73-76). Although eHealth research in this area is limited, it is encouraging to see some new research. Based on two studies included in this scoping review, several user-engagement strategies have been suggested to enhance pediatric care team e-communication. These include (1) developing standard processes for communication and integrating these into e-communication tools; (2) providing care team education and making the standard of e-communication easy to follow; (3) improving use of EHR/etools for communication (e.g., supplying care team members' photos, expertise/roles, contact numbers, and preferred methods of contact in the e-tools); (4) providing incentives for those performing the work/or using the standard $(68,70)$.

\section{Usability}

The usability of e-communication tools and/or standardized e-handover tools were high. Parents and primary care-team members report improvement on their care satisfaction and trust relationship with the care team ( $79 \%$ and $87 \%$, respectively) after use of the tools (68). For e-Handover communication tools, 79\% of care teams reported improvement in team communication, increased satisfaction of care provided to pediatric patients (17\% to $67-87 \%)$ and clinical efficiency (e.g., 68\% reported a significant reduction of paperwork time and/or simplified care processes) $(69,70)$.

\section{DISCUSSION}

The purpose of this paper is to address knowledge gaps in applications of eHealth to promote young children's behavioral and mental health, as well as to understand how eHealth has been applied to broader dissemination of child mental health EBIs. The scoping review method was applied to generate a high-level overview of the eHealth strategies used and evaluated in child mental health contexts. A total of 32 articles (from 30 studies) were selected for this review. Through this review, eHealth applications (what purposes and problems to be solved) and digital- and user-engagement design strategies (how to best design) that have been applied and demonstrated efficacy/ usability/effectiveness in child mental health promotion and prevention were identified and described. eHealth strategies that apply to parents, children, schools, and primary care practices were also identified. Based on our review, we noted several areas where additional eHealth research is needed to develop better approaches to support users in child mental health promotion and prevention both in global and LMIC contexts. In this section, we present research-practice implications for eHealth research that are relevant to LMIC contexts as well as discuss current limitations of the pediatric eHealth field.

\section{Implications for eHealth Development and Design in LMICs}

Four eHealth applications and design lessons can be drawn from this review. First, in identifying eHealth strategies to promote child mental health, three key eHealth design principles were consistently identified across three areas of eHealth applications (parenting, school, and primary care intervention). These include i) core intervention strategies that promote target users' child mental health knowledge and management skills can be developed and adapted from existing EBIs; ii) in promoting e-session/module participation (e.g., increasing adherence, preventing attrition), one or more supporting strategies that motivate participation, promote relationship-connection, or address technical challenges must be in place (e.g., using in-person contact, telephone contact, social media group access, gaming/incentives, automated email, messaging strategies); and iii) in promoting skill practice and behavioral change, consultation, and learning/supporting strategies that match target users' preferences and needs must also in place to ensure the practice changes (e.g., providing consultation support using a group or individual approach).

Second, in designing eHealth that fits LMIC contexts, two lessons can be drawn from the review: i) given limited web/ internet availability in LMICs, the design of eHealth strategies should use mixed approaches by combining offline video-based psychoeducational learning/training (individual or group-based) with mHealth or/and face-to-face support strategies. This might be a feasible model given evidence that either web-based or offline, video-based education approaches can result in promising positive outcomes (61), and that the combination of mHealth and in-person support strategies (in group or individual formats) can be useful and highly acceptable for users with different levels of family risk or child mental health problems $(42,49,56)$; ii) given that evidence has shown similarity in human behavioral change mechanisms across ethnic groups and high- and LMIC populations $(14,77)$, lessons learned from high-income countrybased literature in user-centered design and user-engagement strategies (described in Table 2) are likely to be relevant and applicable to populations in LMICs. However, eHealth design and strategies may need to be tailored to local contexts and to be more thoroughly evaluated.

Third, the potential for applying eHealth strategies as costefficient approaches to address healthcare barriers is high. This scoping review demonstrates that a body of pediatric health research has successfully transported nondigital EBIs to eHealth formats, and has demonstrated the feasibility and effectiveness of this approach in high-income country contexts (24). There are also studies showing evidence of the cost-benefit of eHealth interventions in LMIC settings (78). These findings are encouraging as more EBI literature emerges.

Fourth, from our review, we noted ways that eHealth can be conceptualized and applied as multicomponent/multicontext digital strategies/solutions to promote child mental health. For eHealth to be effective, many eHealth interventions need to integrate multiple components, such as including strategies to promote mental health knowledge, practice and skills. Also, many eHealth interventions need to consider and address needs across multiple contexts, such as considering both family/home (e.g., a child's home), service provider contexts (e.g., primary care, schools), and linkage of both contexts. The multicontext concept is particularly useful in current mental 


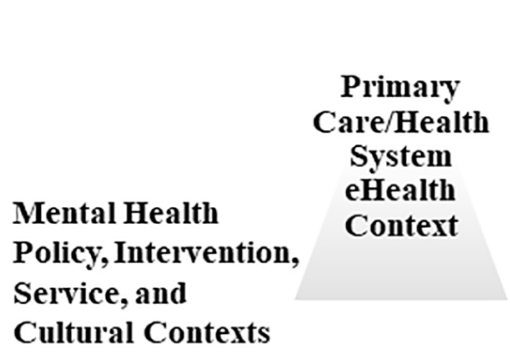

Community/ School eHealth Context
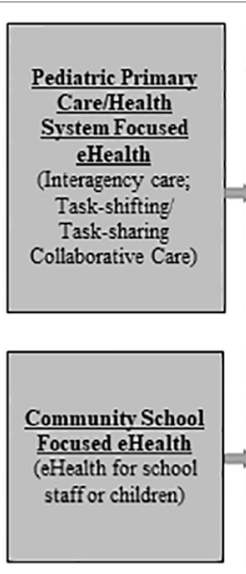

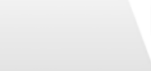

\section{Patient/Family eHealth Context}

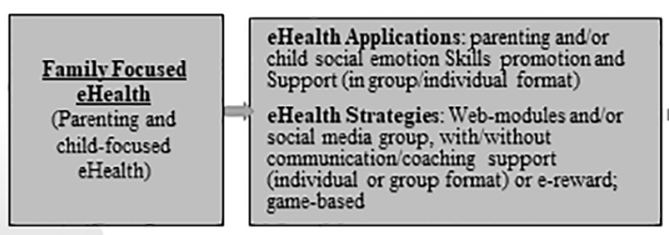

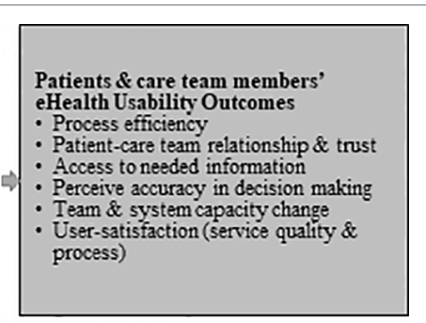

eHealth Applications: eLearning for school staff child mental health or service training; eHealth tools to support two-step triage or behavioral management eHealth Strategies: Web/video education modules, e-discussion forum, e-conference, e-support, e-communication; e-tools for reminder/behavioral prompt/rewards, twostep triage (e-screeningte-service decision)

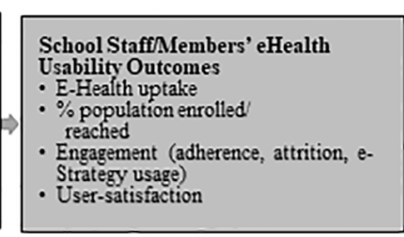

School StaffaMembers' eHealth sability Outcomes E-Health uptake reached

Engagement (adhe User-satisfaction

Parents and/or Children's eHealth Usability Outcomes - E-Health enrollment rate - Usage of e-tech support - Engagement (adhe Strategy usage)

FIGURE 3 | A multicontext framework for child mental and behavioral eHealth. The proposed framework is developed from this eHealth literature synthesis. This framework considers implementation contexts and processes that described in the WHO (2009) Service Pyramid Model (79), and Proctor et al.'s (80) implementation framework.

health intervention service development research given a growing emphasis on linking mental health services from home to communities and health systems, and applying collaborative and team-based care models to integrate mental/behavioral health service in routine primary care and community settings (including the Mental Health Gap Action Program/mhGAP mental health service model suggested by the WHO, which suggests the application of collaborative, task-shifting and tasksharing implementation strategies in the provision of mental health services in LMICs) (73-76).

As an attempt to provide a working framework to guide future eHealth dissemination and implementation research, we summarize our lessons learned in an integrated multicontext framework for child mental and behavioral eHealth (Figure 3). The framework highlights key e-Health applications and strategies at individual/family, school system, and primary care contexts that can be applied to promote child mental health, as well as highlights eHealth usability/implementation outcomes to be considered and measured in future research in order to advance eHealth research and practice.

\section{Limitations and Directions for Future Research}

As a result of this review, we have identified several gaps where additional eHealth research is needed. One, eHealth capacity or system strengthening has rarely been discussed in the eHealth literature or in child mental health research. Given limited eHealth research in LMICs, building eHealth research and system capacity are needed (using the approaches suggested above). eHealth capacity building at the policy/governmental, academic, community, and technology-sector levels will can develop the needed workforce and expertise in designing user- and population-centered eHealth solutions. Two, well-designed high-quality evaluations, such as applying experimental designs to eHealth intervention outcomes studies, are still lacking. More research is needed to map out specific components/approaches of eHealth and associated benefits that can be applied to future development of behavioral health interventions. Three, to advance eHealth research, methodology, and measurement tools for assessing eHealth contexts, target users'/agencies' readiness for eHealth intervention (e.g., technology resources, technology literacy), and eHealth usability and implementation outcomes (e.g., user-engagement level, usercenteredness, usability) need to be further developed. Having better measurement tools and consistent methodology will facilitate cross study comparisons and better mechanism testing research. Four, although telehealth and messaging/texting is not included in this review, given growing applications of these strategies in middleincome countries (81), we suggest a separate scoping review to better understand the applications and stand-alone/unique impacts of these approaches on child health and/or mental health. It will be especially important to consider the impact and applications of telehealth/messaging in those populations that have better access to these eHealth strategies as well as better literacy.

\section{CONCLUSION}

As child mental health issues continue to require complex health service and healthcare policy solutions, it will become 
increasingly important to develop eHealth solutions that consider multiple contexts and integrated multicomponent solutions. This paper provides several new directions to address eHealth programming and methodological gaps related to eHealth research. The scoping review and framework not only provide guidance on how eHealth-related contexts and implementation/usability outcomes can be conceptualized, but also how eHealth mechanisms can be integrated into more robust implementation designs. As has been reiterated, more research is needed to elucidate both cross-setting and multisetting eHealth strategies and mechanisms. In particular, systematic and long-term follow-up research will strengthen understanding of eHealth strategies to advance eHealth implementation effectiveness, sustainability, and system and population-level mental health outcomes.

\section{AUTHOR CONTRIBUTIONS}

K-YH, DL, JN, S-Che, KG, DM, AS, S-Cho, EK, CT, and AM contributed conception and design of the study. K-YH, DL, $\mathrm{JN}$, and S-Che were involved in the acquisition, analysis,

\section{REFERENCES}

1. Murray CJ, Vos T, Lozano R, Naghavi M, Flaxman AD, Michaud C. Disabilityadjusted life years (DALYs) for 291 diseases and injuries in 21 regions, 19902010: a systematic analysis for the Global Burden of Disease Study 2010. Lancet (2013) 380(2197-e223). doi: 10.1016/S0140-6736(12)61689-4

2. Omar MA, Green AT, Bird PK, Mirzoev T, Flisher AJ, Kigozi F, et al. Mental health policy process: a comparative study of Ghana, South Africa, Uganda and Zambia. Int J Ment Health Syst (2010) 4:24. doi: 10.1186/1752-4458-4-24

3. Jamison DT. Disease and mortality in sub-Saharan Africa. Washington, D. C.: World Bank Publications (2006)

4. Ssanyu, R. Mental illness and exclusion: putting mental health on the development agenda in uganda. (2007). https://assets.publishing.service.gov. uk/media/57a08bf9ed915d3cfd0010aa/R8487UPB207.pdf. (Place published: Kampala, Uganda)

5. McCoy DC, Peet ED, Ezzati M, Danaei G, Black MM, Sudfeld CR, et al. Early childhood developmental status in low-and middle-income countries: national, regional, and global prevalence estimates using predictive modeling. PloS Med (2016) 13(6):e1002034. doi: 10.1371/journal.pmed.1002233

6. WHO. Social determinants of health and well-being among young people: Health behaviour in school-aged children (HBSC) study-international report from the 2009-2010 survey. WHO (2012) Copenhagen, Denmark. Available from: http:// www.euro.who.int/:data/assets/pdf_file/0007/167281/E96444_part1.pdf.

7. WHO. Closing the gap in a generation: Health equity through action on the social determinants of health. Report from the commission on social determinants of health (2008 April 2012). Available from: http://apps.who. int/iris/bitstream/10665/43943/1/9789241563703_eng.pdf.

8. Dunn JR, Dyck I. Social determinants of health in Canada's immigrant population: results from the National Population Health Survey. Soc Sci Med (2000) 51:1573-93. doi: 10.1016/S0277-9536(00)00053-8

9. CDC. Establishing a Holistic Framework to Reduce Inequities in HIV, Viral Hepatitis, STDs, and Tuberculosis in the United States (2010 Oct 2012). Available from: http://www.cdc.gov/socialdeterminants/.

10. Huang KY, Bornheimer LA, Dankyi E, de-Graft Aikins A. Parental wellbeing, parenting and child development in Ghanaian families with young children. Child Psychiatry Hum Dev (2018) 49(5):833-41. doi: 10.1007/ s10578-018-0799-3 and interpretation of data. $\mathrm{K}-\mathrm{YH}$ wrote the first draft of the manuscript. K-YH, DL, S-Che, KG, AS, and AM contributed to manuscript writing. All authors contributed to manuscript revision, read, and approved the submitted version.

\section{FUNDING}

This research was supported by grant NIH/NCATS 1UL1TR001445 from the National Institutes of Health (NIH), and R21 MH110001-01 and U19MH110001-01 from the National Institute of Mental Health (NIMH). The reviews and opinions expressed in this article are solely those of the authors and do not necessarily represent the views of NIH. This review relied on publicly available documents and, therefore, was exempt from Institutional Review Board determination.

\section{SUPPLEMENTARY MATERIAL}

The Supplementary Material for this article can be found online at: https://www.frontiersin.org/articles/10.3389/fpsyt.2019.00806/ full\#supplementary-material

11. Huang K-Y, Abura G, Theise R, Nakigudde J. Parental depression and associations with parenting and children's physical and mental health in SubSaharan African settings. Child Psychiatry Hum Dev (2017) 48:517-27. doi: 10.1007/s10578-016-0679-7

12. Huang K-Y, Cheng S, Theise R. School contexts as social determinants of Health: current practices and impliactions for future public health practice. Public Health Rep (2013) 128:21-8. doi: 10.1177/00333549131286S304

13. Huang K-Y, Calzada E, Kamboukos D, Rhule D, Cullen KW, Cheng S, et al. Applying public health frameworks to advance the promotion of mental health among Asian American children. Asian Am J Psychol (2014) 5:14552. doi: 10.1037/a0036185

14. Huang KY, Nakigudde J, Ruhule D, Gumikiriza JL, Abura G, Kolawole B, et al. Transportability of an evidence-based early childhood intervention in a low-income African Country: results of a cluster randomized controlled implementation study. Prev Sci (2017) 18:964-75. doi: 10.1007/ s11121-017-0822-0

15. Labrique AB, Vasudevan L, Kochi E, Fabricant R, Mehl G. mHealth innovations as health system strengthening tools: 12 common applications and a visual framework. Global Health: Sci Pract (2013) 1:161-71. doi: 10.9745/GHSP-D-13-00031

16. Davies EB, Morriss R, Glazebrook C. Computer-delivered and web-based interventions to improve depression, anxiety, and psychological well-being of university students: a systematic review and meta-analysis. J Med Internet Res (2014) 16(5):e130. doi: 10.2196/jmir.3142

17. WHO. Atlas of eHealth country profiles: based on the findings of the second global survey on eHealth (2011) [cited 2019 April 2019]. Available from: https://apps.who.int/iris/bitstream/handle/10665/44502/9789241564168_ eng.pdf? sequence $=1$

18. WHO. eHealth solutions in the Afircan Region: Current Context and Perspectives: WHO (2010) [cited 2014 April]. Available from: www.afro. who.int/index.php?option=com_docman\&task=doc_download\&gid $=5623$.

19. Free C, Phillips G, Watson L, Galli L, Felix L, Edwards P, et al. The effectiveness of mobile-health technologies to improve health care service delivery processes: a systematic review and meta-analysis. PloS Med (2013) 10(1):e1001363. doi: 10.1371/journal.pmed.1001363

20. WHO. Global Observaotry for eHealth: WHO (2019) [cited 2019 April]. Available from: https://www.who.int/goe/en/. 
21. WHO. National eHealth Strategy Toolkit (2012). Available from: https:// apps.who.int/iris/bitstream/handle/10665/75211/9789241548465_eng. pdf? sequence $=1$.

22. WHO. Atlas of eHealth country profiles 2015: The use of eHealth in support of universal health coverage (2016) [cited 2019 April 2019]. Available from: https://www.who.int/goe/publications/atlas_2015/en/.

23. WHO. eHealth and innovation in women's and children's health: a baseline review (2014). Available from: https://apps.who.int/iris/bitstream/ handle/10665/111922/9789241564724_eng.pdf?sequence $=1$.

24. Barros L, Greffin K. Supporting health-related parenting: a scoping review of programs assisted by the internet and related technologies. Estudos Psicologia (Campinas) (2017) 34(3):331-44. doi: 10.1590/1982-02752017000300002

25. Bervell B, Al-Samarraie H. A comparative review of mobile health and electronic health utilization in sub-Saharan African countries. Soc Sci Med (1982) (2019) 232:1-16. doi: 10.1016/j.socscimed.2019.04.024

26. Breitenstein SM, Gross D, Christophersen R. Digital delivery methods of parenting training interventions: a systematic review. Worldviews Evid Based Nurs (2014) 11:168-76. doi: 10.1111/wvn.12040

27. Kim H, Xie B. Health literacy in the eHealth era: a systematic review of the literature. Patient Educ Couns (2017) 100(6):1073-82. doi: 10.1016/j. pec.2017.01.015

28. Ramukumba MM, Hagglund M. "I feel like a nurse and my clients learn more": mHealth, Capacity Building and Empowerment in Community Based Care. Studies Health Technol Inf (2019) 265:195-200. doi: 10.3233/ shti190163

29. Eden R, Burton-Jones A, Scott I, Staib A, Sullivan C. Effects of eHealth on hospital practice: synthesis of the current literature. Aust Health Rev: Publ Aust Hosp Assoc (2018) 42(5):568-78. doi: 10.1071/ah17255

30. Farkas C, Solodiuk L, Taddio A, Franck L, Berberich FR, LoChiatto J, et al. Publicly available online educational videos regarding pediatric needle pain: a scoping review. Clin J Pain (2015) 31(6):591-8. doi: 10.1097/ ajp.0000000000000197

31. Poushter J. Smartphone ownership and internet usage continues to climb in emerging economies. (2016). Pew Research Center, 22, 1-44. https:// www.pewresearch.org/global/wp-content/uploads/sites/2/2016/02/pew_ research_center_global_technology_report_final_february_22_2016.pdf

32. Poushter J, Oates R. Cell phones in Africa: communication lifeline: Pew Research Center. (2015). https://www.pewresearch.org/global/wp-content/ uploads/sites/2/2015/04/Pew-Research-Center-Africa-Cell-Phone-ReportFINAL-April-15-2015.pdf

33. Arksey H, O’Malley L. Scoping studies: towards a methodological framework. Int J Soc Res Method (2005) 8(1):19-32. doi: 10.1080/1364557032000119616

34. Tricco AC, Lillie E, Zarin W, O’Brien KK, Colquhoun H, Levac D, et al. PRISMA extension for scoping reviews (PRISMA-ScR): checklist and explanation. Ann Intern Med (2018) 169(7):467-73. doi: 10.7326/M18-0850

35. Nelson EL, Sharp S. A review of pediatric telemental health. Pediatr Clin North Am (2016) 63(5):913-31. doi: 10.1016/j.pcl.2016.06.011

36. Mejia A, Calam R, Sanders MR. A review of parenting programs in developing countries: opportunities and challenges for preventing emotional and behavioral difficulties in children. Clin Child Fam Psychol Rev (2012) 15(2):163-75. doi: 10.1007/s10567-012-0116-9

37. Gardner F, Montgomery P, Knerr W. Transporting evidence-based parenting programs for child problem behavior (Age 3-10) between countries: systematic review and meta-analysis. J Clin Child Adolesc Psychol (2016) 45(6):749-62. doi: 10.1080/15374416.2015.1015134

38. Baumann AA, Powell BJ, Kohl PL, Tabak RG, Penalba V, Proctor EE, et al. Cultural adaptation and implementation of evidence-based parent-training: a systematic review and critique of guiding evidence. Child Youth Serv Rev (2015) 53:113-20. doi: 10.1016/j.childyouth.2015.03.025

39. Coker TR, Porras-Javier L, Zhang L, Soares N, Park C, Patel A, et al. A telehealthenhanced referral process in pediatric primary care: a cluster randomized trial. Pediatrics (2019) 143(3):e20182738. doi: 10.1542/peds.2018-2738

40. Malas N, Klein E, Tengelitsch E, Kramer A, Marcus S, Quigley J. Exploring the telepsychiatry experience: primary care provider perception of the Michigan Child Collaborative Care (MC3) Program. Psychosomatics (2019) 60(2):179-89. doi: 10.1016/j.psym.2018.06.005

41. Tolou-Shams M, Yonek J, Galbraith K, Bath E. Text messaging to enhance behavioral health treatment engagement among justice-involved youth: qualitative and user testing study. JMIR mHealth uHealth (2019) 7(4):e10904. doi: $10.2196 / 10904$

42. Enebrink P, Hogstrom J, Forster M, Ghaderi A. Internet-based parent management training: a randomized controlled study. Behav Res Ther (2012) 50(4):240-9. doi: 10.1016/j.brat.2012.01.006

43. Edwards-Gaura A, Whitaker D, Self-Brown S. Can social networking be used to promote engagement in child maltreatment prevention programs? Two pilot studies. Western J Emergency Med (2014) 15(5):575-81. doi: 10.5811/ westjem.2014.4.21413

44. Choi H, Kim S, Ko H, Kim Y, Park C. Development and preliminary evaluation of culturally specific web-based intervention for parents of adolescents. J Psychiatr Ment Health Nurs (2016) 23(8):489-501. doi: 10.1111/jpm. 12327

45. Sourander A, McGrath PJ, Ristkari T, Cunningham C, Huttunen J, LingleyPottie $\mathrm{P}$, et al. Internet-assisted parent training intervention for disruptive behavior in 4-year-old children: a randomized clinical trial. JAMA Psychiatry (2016) 73(4):378-87. doi: 10.1001/jamapsychiatry.2015.3411

46. DeHoff BA, Staten LK, Rodgers RC, Denne SC. The role of online social support in supporting and educating parents of young children with special health care needs in the United States: a scoping review. J Med Internet Res (2016) 18(12):e333. doi: 10.2196/jmir.6722

47. Breitenstein SM, Schoeny M, Risser H, Johnson T. A study protocol testing the implementation, efficacy, and cost effectiveness of the ezParent program in pediatric primary care. Contemp Clin trials (2016) 50:229-37. doi: 10.1016/j.cct.2016.08.017

48. Breitenstein SM, Brager J, Ocampo EV, Fogg L. Engagement and adherence with ezPARENT, an mHealth parent-training program promoting child wellbeing. Child Maltreat (2017) 22(4):295-304. doi: 10.1177/10775559517725402

49. Love SM, Sanders MR, Turner KM, Maurange M, Knott T, Prinz R, et al Social media and gamification: engaging vulnerable parents in an online evidence-based parenting program. Child Abuse Negl (2016) 53:95-107. doi: 10.1016/j.chiabu.2015.10.031

50. Baker S, Sanders MR, Turner KMT, Morawska A. A randomized controlled trial evaluating a low-intensity interactive online parenting intervention, Triple P Online Brief, with parents of children with early onset conduct problems. Behav Res Ther (2017) 91:78-90. doi: 10.1016/j. brat.2017.01.016

51. Baker S, Sanders MR. Predictors of program use and child and parent outcomes of a brief online parenting intervention. Child Psychiatry Hum Dev (2017) 48(5):807-17. doi: 10.1007/s10578-016-0706-8

52. Hemdi A, Daley D. The effectiveness of a psychoeducation intervention delivered via WhatsApp for mothers of children with Autism Spectrum Disorder (ASD) in the Kingdom of Saudi Arabia: a randomized controlled trial. Child: Care Health Dev (2017) 43(6):933-41. doi: 10.1111/cch.12520

53. Morgan AJ, Rapee RM, Salim A, Goharpey N, Tamir E, McLellan LF, et al. Internet-delivered parenting program for prevention and early intervention of anxiety problems in young children: randomized controlled trial. $J$ Am Acad Child Adolesc Psychiatry (2017) 56(5):417-25. doi: 10.1016/j. jaac.2017.02.010

54. Owen DA, Griffith N, Hutchings J. Evaluation of the COPING parent online universal programme: study protocol for a pilot randomised controlled trial. BMJ Open (2017) 7(4):e013381. doi: 10.1136/bmjopen-2016-013381

55. Tully LA, Piotrowska PJ, Collins DAJ, Mairet KS, Hawes DJ, Kimonis ER, et al. Study protocol: evaluation of an online, father-inclusive, universal parenting intervention to reduce child externalising behaviours and improve parenting practices. BMC Psychol (2017) 5(1):21. doi: 10.1186/ s40359-017-0188-x

56. Day JJ, Sanders MR. Do parents benefit from help when completing a selfguided parenting program online? A randomized controlled trial comparing triple P Online with and without telephone support. Behav Ther (2018) 49(6):1020-38. doi: 10.1016/j.beth.2018.03.002

57. Fernando LMN, Sim WH, Jorm AF, Rapee R, Lawrence KA, Yap MBH. Parenting Resilient Kids (PaRK), an online parenting program to prevent anxiety and depression problems in primary school-aged children: study protocol for a randomised controlled trial. Trials (2018) 19(1):236. doi: 10.1186/s13063-018-2605-8

58. Sanchez R, Brown E, Kocher K, DeRosier M. Improving children's mental health with a digital social skills development game: a randomized controlled 
efficacy trial of adventures aboard the S.S. GRIN. Games Health J (2017) 6(1):19-27. doi: 10.1089/g4h.2015.0108

59. Warnestal P, Svedberg P, Lindberg S, Nygren JM. Effects of using child personas in the development of a digital peer support service for childhood cancer survivors. J Med Internet Res (2017) 19(5):e161. doi: 10.2196/ jmir.7175

60. Stasiak K, Merry S, Frampton C, Moor S. Delivering solid treatments on shaky ground: Feasibility study of an online therapy for child anxiety in the aftermath of a natural disaster. Psychother Res (2018) 28(4):643-53. doi: 10.1080/10503307.2016.1244617

61. Pereira CA, Wen CL, Miguel EC, Polanczyk GV. A randomised controlled trial of a web-based educational program in child mental health for schoolteachers. Eur Child Adolesc Psychiatry (2015) 24(8):931-40. doi: 10.1007/s00787-014-0642-8

62. Schuck S, Emmerson N, Ziv H, Collins P, Arastoo S, Warschauer M, et al. Designing an iPad app to monitor and improve classroom behavior for children with ADHD: iSelfControl feasibility and pilot studies. PloS One (2016) 11(10):e0164229. doi: 10.1371/journal.pone.0164229

63. Bezem J, Heinen D, Reis R, Buitendijk SE, Numans ME, Kocken PL. Improving access to school health services as perceived by school professionals. BMC Health Serv Res (2017) 17(1):743. doi: 10.1186/s12913-017-2711-4

64. Ahlers-Schmidt CR, Nguyen M. Parent intention to use a patient portal as related to their children following a facilitated demonstration. Telemedicine $J$ e-health: official J Am Telemedicine Assoc (2013) 19(12):979-81. doi: 10.1089/ tmj.2013.0041

65. Fothergill KE, Gadomski A, Solomon BS, Olson AL, Gaffney CA, Dosreis $\mathrm{S}$, et al. Assessing the impact of a web-based comprehensive somatic and mental health screening tool in pediatric primary care. Academic Pediatr (2013) 13(4):340-7. doi: 10.1016/j.acap.2013.04.005

66. Glascoe FP. Evidence-based early detection of developmental-behavioral problems in primary care: What to expect and how to do it. J Pediatr Health Care (2015) 29(1):46-53. doi: 10.1016/j.pedhc.2014.06.005

67. O'Connor S, Devlin AM, McGee-Lennon M, Bouamrane MM, O'Donnell CA, Mair FS. Factors affecting participation in the eRedBook: a personal child health record. Stud Health Technol Inf (2016) 225:971-2. doi: 10.3233/978-1-61499-658-3-971

68. Singh A, Rhee KE, Brennan JJ, Kuelbs C, El-Kareh R, Fisher ES. Who's my doctor? Using an electronic tool to improve team member identification on an inpatient pediatrics team. Hosp Pediatr (2016) 6(3):157-65. doi: 10.1542/ hpeds.2015-0164

69. Cheng DR, Liddle J, Mailes E, South M. Impact of an integrated electronic handover tool on pediatric junior medical staff (JMS) handover. Int J Med Inf (2017) 108:92-6. doi: 10.1016/j.ijmedinf.2017.10.009

70. Destino LA, Dixit A, Pantaleoni JL, Wood MS, Pageler NM, Kim J, et al. Improving communication with primary care physicians at the time of hospital discharge. Joint Commission J Qual Patient Saf (2017) 43(2):80-8. doi: 10.1016/j.jcjq.2016.11.005

71. Fiks AG, Mayne SL, Michel JJ, Miller J, Abraham M, Suh A, et al. DistanceLearning, ADHD quality improvement in primary care: a clusterrandomized trial. J Dev Behav Pediatr: JDBP (2017) 38(8):573-83. doi: 10.1097/dbp.0000000000000490
72. Latif A, Carter T, Rychwalska-Brown L, Wharrad H, Manning J. Co-producing a digital educational programme for registered children's nurses to improve care of children and young people admitted with selfharm. J Child Health Care: Professionals Working Children Hosp Community (2017) 21(2):191-200. doi: 10.1177/1367493517697853

73. Bergman D, Plsek P, Saunders M. A high-performing system for well-child care: a vision for the future. (2006). Available from: http:// www.commonwealthfund.org/publications/fund-reports/2006/oct/ a-high-performing-system-for-well-child-care-a-vision-for-the-future.

74. Molnar BE, Lees KE, Roper K, Byars N, Mendez-Penate L, Moulin C, et al. Enhancing early childhood mental health primary care services: evaluation of MA Project LAUNCH. Maternal Child Health J (2018) 22(10):1502-10. doi: 10.1007/s10995-018-2548-4

75. Campo JV, Geist R, Kolko DJ. Integration of pediatric behavioral health services in primary care: improving access and outcomes with collaborative care. Can J Psychiatry (2018) 63(7):432-8. doi: 10.1177/0706743717751668

76. World Health Organization. mhGAP Intervention Guide- Version 2.0 for mental, neurological and substance use disorders in non-specialized health settings (2016). Available from: http://www.who.int/mental_health/mhgap/ mhGAP_intervention_guide_02/en/.

77. Giusto A, Puffer E. A systematic review of interventions targeting men's alcohol use and family relationships in low- and middle-income countries. Global Ment Health (Cambridge England) (2018) 5:e10. doi: 10.1017/gmh.2017.32

78. Sureshkumar K, Murthy GV, Munuswamy S, Goenka S, Kuper H. 'Care for Stroke', a web-based, smartphone-enabled educational intervention for management of physical disabilities following stroke: feasibility in the Indian context. BMJ Innovations (2015) 1(3):127-36. doi: 10.1136/ bmjinnov-2015-000056

79. WHO. Improving Health Systems and Services for Mental Health (2009). Available from: http://apps.who.int/iris/bitstream/10665/44219/1/978924 1598774_eng.pdf.

80. Proctor EK, Landsverk J, Aarons G, Chambers D, Glisson C, Mittman B. Implementation research in mental health services: an emerging science with conceptual, methodological, and training challenges. Adm Policy Ment Health (2009) 36:24-34. doi: 10.1007/s10488-008-0197-4

81. Dinesen B, Nonnecke B, Lindeman D, Toft E, Kidholm K, Jethwani K, et al. Personalized telehealth in the future: a global research agenda. J Med Internet Res (2016) 18(3):e53. doi: 10.2196/jmir.5257

Conflict of Interest: The authors declare that the research was conducted in the absence of any commercial or financial relationships that could be construed as a potential conflict of interest.

Copyright (®) 2019 Huang, Lee, Nakigudde, Cheng, Gouley, Mann, Schoenthaler, Chokshi, Kisakye, Tusiime and Mendelsohn. This is an open-access article distributed under the terms of the Creative Common Attribution License (CC BY). The use, distribution or reproduction in other forums is permitted, provided the original author(s) and the copyright owner(s) are credited and that the original publication in this journal is cited, in accordance with accepted academic practice. No use, distribution or reproduction is permitted which does not comply with these terms. 